\title{
PEANO ARITHMETIC AND HYPER-RAMSEY LOGIC
}

\author{
JAMES H. SCHMERL ${ }^{1}$
}

\begin{abstract}
It is known that $\operatorname{PA}\left(Q^{2}\right)$, Peano arithmetic in a language with the Ramsey quantifier, is complete and compact and that its first-order consequences are the same as those of $\Pi_{1}^{1}-\mathrm{CA}_{0}$. A logic $\mathscr{H} \mathscr{R}_{\omega}$, called hyper-Ramsey logic, is

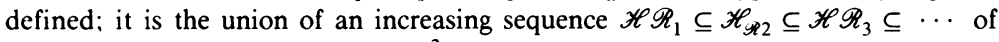
sublogics, and $\mathscr{H} \mathscr{R}_{1}$ contains $L\left(Q^{2}\right)$. It is proved that PA $\left(\mathscr{H}_{n} \mathscr{R}_{n}\right)$, which is Peano arithmetic in the context of $\mathscr{H} \mathscr{R}_{n}$, has the same first-order consequences as $\mathrm{II}_{n}^{1}-\mathrm{CA}_{0}$. A by-product and ingredient of the proof is, for example, the existence of a model of CA having the form $(\mathscr{N}, \operatorname{Class}(\mathscr{N}))$.
\end{abstract}

The extension of first-order Peano arithmetic (PA) by its augmentation with Ramsey quantifiers was suggested by Macintyre [4] in reaction to the finite combinatorial incompletenesses of the Paris-Kirby-Harrington type. His intention was to give the Ramsey quantifiers a "Cantorian" interpretation: if $\mathscr{N}$ is a model of PA, then $\mathscr{N} \vDash Q^{n} x_{1} x_{2} \cdots x_{n} \phi\left(x_{1}, x_{2}, \ldots, x_{n}\right)$ iff there is an unbounded $X \subseteq N$ such that $\mathscr{N} \vDash \phi\left(a_{1}, a_{2}, \ldots, a_{n}\right)$ for all distinct $a_{1}, a_{2}, \ldots, a_{n} \in X$. Macintyre refers to the theory Ind, which is essentially PA together with the induction scheme for all Ramsey formulas in the language of PA. The theory $\operatorname{PA}\left(Q^{2}\right)$, which consists of those sentences of Ind involving just the 2-place Ramsey quantifier $Q^{2}$, was precisely axiomatized in [8]. Both the model-theoretic and proof-theoretic properties of $\operatorname{PA}\left(Q^{2}\right)$ have been well analyzed in the two papers [4 and 8], with which the reader should have some familiarity. It was stated in [8] that $\operatorname{PA}\left(Q^{2}\right)$ and Ind are "essentially equivalent." If $\S 3$ we discuss the relationship between $\operatorname{PA}\left(Q^{2}\right)$ and Ind, and also the role in Peano arithmetic of other quantifiers of the Ramsey type.

Even though it is a theory in an extended first-order logic, $\operatorname{PA}\left(Q^{2}\right)$ is naturally a fragment of second-order arithmetic with the formula $Q^{2} x x^{\prime} \phi\left(x, x^{\prime}\right)$ being an abbreviation for

$$
\exists X\left[\forall y \exists z(z>y \wedge z \in X) \wedge \forall x x^{\prime} \in X\left(x \neq x^{\prime} \rightarrow \phi\left(x, x^{\prime}\right)\right)\right] .
$$

In this way, two-sorted structures of the form $(\mathscr{N}, \mathscr{X})$, where $\mathscr{X} \subseteq \mathscr{P}(N)$, the power set of $N$, are appropriate for Ramsey logic, with strong models becoming identified with the full second-order structures $(\mathscr{N}, \mathscr{P}(N))$. Macintyre suggests in $\S 5.6$ of [4]

Received by the editors August 17, 1984.

1980 Mathematics Subject Classification. Primary 03H15; Secondary 03C80, 30F35, 03C85.

Key words and phrases. Peano arithmetic, second-order theories of arithmetic, Ramsey quantifier, ramified analytical hierarchy, hyper-Ramsey logic.

${ }^{1}$ This research was supported in part by NSF Grant No. 8301603. 
that: "It would be interesting to find other fragments of second-order arithmetic with nonstandard models in the intended (Cantorian) semantics."

In this paper we respond to Macintyre's suggestion, obtaining other quite natural fragments of second-order arithmetic, by considering what we call hyper-Ramsey logic. Although its syntax is second-order, hyper-Ramsey logic is best thought of as an extended first-order logic. Peano arithmetic, in the context of hyper-Ramsey logic, is denoted by $\operatorname{PA}\left(\mathscr{H} \mathscr{R}_{\omega}\right)$. It will be seen that $\operatorname{PA}\left(\mathscr{H} \mathscr{R}_{\omega}\right)$ corresponds to the full comprehension scheme in the same way that $\operatorname{PA}\left(Q^{2}\right)$ corresponds to $\Pi_{1}^{1}-\mathrm{CA}_{0}$. In fact, sharper results are obtained by showing that $\operatorname{PA}\left(\mathscr{H}_{\mathscr{R}_{\omega}}\right)$ is the union of an increasing sequence $\left\langle\operatorname{PA}\left(\mathscr{H} \mathscr{R}_{n}\right): n<\omega\right\rangle$ of subtheories, each $\mathrm{PA}\left(\mathscr{H}_{\mathscr{R}_{n}}\right)$ roughly corresponding to $\Pi_{n}^{1}-\mathrm{CA}_{0}$ and $\operatorname{PA}\left(\mathscr{H} \mathscr{R}_{1}\right)$ having $\operatorname{PA}\left(Q^{2}\right)$ as a subtheory.

The contents of this paper are outlined as follows. A review of some second-order theories of arithmetic is presented in $\S 1$. That section concludes with Theorem 1.5 in which it is shown that in the models $(\mathscr{N}, \mathscr{X})$ of some rather weak second-order theories of arithmetic, a modification of the ramified analytical hierarchy can be used to obtain models $\left(\mathscr{N}, \mathscr{X}^{\prime}\right)$, with $\mathscr{X}^{\prime} \subseteq \mathscr{X}$, of apparently stronger theories. In $\S 2$ we give some new results, which should be of independent interest, on the model theory of second-order theories of arithmetic. The objects studied in this section are natural models; these are structures of the form $(\mathscr{N}, \mathscr{X})$, where $\mathscr{X}$ is the collection of all classes of $\mathscr{N}$. In $\S 3$ we discuss Peano arithmetic with other quantifiers of the Ramsey type, most notably $Q^{1,1}$. We show that although $\operatorname{PA}\left(Q^{1,1}\right)$ and $\operatorname{PA}\left(Q^{2}\right)$ have the same first-order consequences, there are models of $\operatorname{PA}\left(Q^{1,1}\right)$ which are not models of $\operatorname{PA}\left(Q^{2}\right)$. Hyper-Ramsey logic is finally introduced in $\S 4$, and Peano arithmetic for this logic, $\operatorname{PA}\left(\mathscr{H} \mathscr{R}_{\omega}\right)$, is defined. Then in $\S 5$ the construction of models of $\operatorname{PA}\left(\mathscr{H} \mathscr{R}_{\omega}\right)$ is presented.

The results of this paper were announced in [9]. A large amount of credit is due Matt Kaufmann who turned a vague idea into the proof of Lemma 5.2. We are greatly indebted to him for this proof and also for many illuminating discussions on the subject of this paper.

1. Second-order arithmetic. The first part of this section is concerned with a review of some second-order theories which are subtheories of true second-order arithmetic. Structures appropriate for such theories have the form $(\mathscr{N}, \mathscr{X})$, where $\mathscr{N} \vDash$ PA and $\mathscr{X} \subseteq \mathscr{P}(N)$. These structures are two-sorted structures with first-order variables (written in lower case) referring to elements of $N$ and second-order variables (written in upper case) referring to elements of $\mathscr{X}$.

In the second part of this section, consisting of Theorem 1.5 and its proof, we show that if $(\mathscr{N}, \mathscr{X})$ is a model of what we call $\Lambda_{n}^{1}-\mathrm{CA}_{0}$ (which is a sort of parameter-free version of $\left.\Pi_{n}^{1}-\mathrm{CA}_{0}\right)$, then, by a modification of the construction of the ramified analytical hierarchy, there exists $\mathscr{X}^{\prime} \subseteq \mathscr{X}$ such that $\left(\mathscr{N}, \mathscr{X}^{\prime}\right) \vDash \Pi_{n}^{1}$-CA 0 .

We begin with some syntax. As usual, we let $\Pi_{0}^{1}=\Sigma_{0}^{1}$ be the class of arithmetic formulas; these are formulas having no second-order quantifiers. Then for $n<\omega$ we inductively define $\Pi_{n+1}^{1}$ and $\Sigma_{n+1}^{1}$ : a formula $\phi$ is in $\Pi_{n+1}^{1}$ iff it is of the form $\forall X \psi$, where $\psi \in \Sigma_{n}^{1}$, and it is in $\Sigma_{n+1}^{1}$ iff it is of the form $\exists X \psi$, where $\psi \in \Pi_{n}^{1}$. Often, we 
shall say a formula is in $\Pi_{n}^{1}$ or $\Sigma_{n}^{1}$ when it is only equivalent to one in $\Pi_{n}^{1}$ or $\Sigma_{n}^{1}$. In particular, we will assume that $\Sigma_{n}^{1} \cup \Pi_{n}^{1} \subseteq \Sigma_{n+1}^{1} \cap \Pi_{n+1}^{1}$.

We will make use of some standard pairing function $x, y \mapsto\langle x, y\rangle$, and then let $(X)_{x}=\{y:\langle x, y\rangle \in X\}$. We will also let $\langle X, Y\rangle=Z$, where $(Z)_{0}=X,(Z)_{1}=Y$, and $(Z)_{z}=\varnothing$ for $z \geqslant 2$.

The following are the basic axioms and axiom schemes of second-order arithmetic.

Induction axiom (IA): $\forall X[0 \in X \wedge \forall x(x \in X \rightarrow x+1 \in X) \rightarrow \forall x(x \in X)]$.

$\Pi_{n}^{1}$-comprehension scheme: $\exists X \forall x(x \in X \leftrightarrow \phi(x))$, where $\phi(x)$ is $\Pi_{n}^{1}$.

$\Delta_{n}^{1}$-comprehension scheme: $\forall x(\phi(x) \leftrightarrow \psi(x)) \rightarrow \exists X \forall x(x \in X \leftrightarrow \phi(x))$, where both $\phi(x)$ and $\psi(x)$ are $\Pi_{n}^{1}$.

$\sum_{n+1}^{1}$-choice scheme: $\forall x \exists X \phi(x, X) \rightarrow \exists X \forall x \phi\left(x,(X)_{x}\right)$, where $\phi(x, X)$ is $\Pi_{n}^{1}$.

$\Sigma_{n+1}^{1}$-collection scheme: $\forall x \exists X \phi(x, X) \rightarrow \exists X \forall x \exists y \phi\left(x,(X)_{y}\right)$, where $\phi(x, X)$ is $\Pi_{n}^{1}$.

In the four schemes above, the $\Pi_{n}^{1}$ formulas $\phi$ and $\psi$ may have undisplayed firstand second-order variables. The $\Pi_{0}^{1}$-comprehension scheme is also called the arithmetic comprehension scheme.

The basic theories, which are all subtheories of true second-order arithmetic, are the following ones:

$\mathrm{ACA}_{0}=\mathrm{PA}+\mathrm{IA}+$ the arithmetic comprehension scheme;

$\Pi_{n+1}^{1}-\mathrm{CA}_{0}=\mathrm{ACA}_{0}+$ the $\Pi_{n+1}^{1}$-comprehension scheme;

$\Delta_{n+1}^{1}-\mathrm{CA}_{0}=\mathrm{ACA}_{0}+$ the $\Delta_{n+1}^{1}$-comprehension scheme;

$\Sigma_{n+1}^{1}-\mathrm{AC}_{0}=\mathrm{ACA}_{0}+$ the $\Sigma_{n+1}^{1}$-choice scheme;

$\Sigma_{n+1}^{1}$-Coll $=\mathrm{ACA}_{0}+$ the $\Sigma_{n+1}^{1}$-collection scheme.

For convenience we let $\Pi_{0}^{1}-\mathrm{CA}_{0}=\Sigma_{0}^{1}-\mathrm{AC}_{0}=\Sigma_{0}^{1}$-Coll $=\mathrm{ACA}_{0}$. We also let CA $=\Pi_{\omega}^{1}-\mathrm{CA}_{0}=\bigcup_{n<\omega} \Pi_{n}^{1}-\mathrm{CA}_{0}$ and $\mathrm{AC}=\Sigma_{\omega}^{1}-\mathrm{AC}_{0}=\bigcup_{n<\omega} \Sigma_{n}^{1}-\mathrm{AC}_{0}$.

The following standard lemma is well known and easily proved.

Lemma 1.1. Suppose $(\mathscr{N}, \mathscr{X}) \vDash \mathrm{ACA}_{0}$ and $n<\omega$. Then

$$
(\mathscr{N}, \mathscr{X}) \vDash \Sigma_{n+1}^{1}-\mathrm{AC}_{0} \Rightarrow(\mathscr{N}, \mathscr{X}) \vDash \Sigma_{n+1}^{1}-\text { Coll } \Rightarrow(\mathscr{N}, \mathscr{X}) \vDash \Pi_{n}^{1}-\mathrm{CA}_{0}
$$

and

$$
(\mathscr{N}, \mathscr{X}) \vDash \Delta_{n+1}^{1}-\mathrm{CA}_{0}+\Sigma_{n+1}^{1}-\text { Coll } \Rightarrow(\mathscr{N}, \mathscr{X}) \vDash \Sigma_{n+1}^{1}-\mathrm{AC}_{0} .
$$

We will make use of another hierarchy of formulas.

DEFINITION 1.2. For a second-order formula $\phi$, its level $\lambda(\phi)$ is the number of distinct quantified second-order variables occurring in $\phi$.

For example, if $\phi$ is $\Pi_{n}^{1}$, then $\lambda(\phi) \leqslant n$. Boolean combinations of formulas of level $n$ have level $n$ (provided, of course, that the quantified second-order variables in the constituent formulas are replaced by variables from some fixed pool of $n$ variables). If $\lambda(\phi) \leqslant n$, then $\exists x \phi$ and $\forall x \phi$ both have level at most $n$. There is a certain type of nesting that can be done with formulas preserving their levels. Let $\phi$ and $\psi$ be formulas such that $\lambda(\phi), \lambda(\psi) \leqslant n$, with $X$ a free variable of $\phi$ and $x$ a free variable of $\psi$. Then the formula $\forall X[\forall x(x \in X \leftrightarrow \psi(x)) \rightarrow \phi]$ is equivalent to a 
formula of level $n$ which is derived from $\phi$ by replacing each occurrence of an atomic formula $u \in X$ by $\psi(u)$.

Let $\Lambda_{n}^{1}$ be the set of formulas $\phi$ such that $\lambda(\phi) \leqslant n$. The following less familiar scheme will be useful in this paper.

$\Lambda_{n}^{1}$-comprehension scheme: $\exists X \forall x(x \in X \leftrightarrow \phi(x))$, where $\phi(x)$ is a formula in $\Lambda_{n}^{1}$ with no free second-order variables.

In a structure $(\mathscr{N}, \mathscr{X})$ we say that a set $A \in \mathscr{X}$ is $\Lambda_{n}^{1}$-definable if it is definable by means of a $\Lambda_{n}^{1}$ formula with no second-order parameters. Even though no secondorder parameters are allowed in the $\Lambda_{n}^{1}$-comprehension scheme, nothing is gained by allowing parameters which are themselves $\Lambda_{n}^{1}$-definable.

There is a theory corresponding to the $\Lambda_{n}^{1}$-comprehension scheme:

$$
\Lambda_{n}^{1}-\mathrm{CA}_{0}=\mathrm{ACA}_{0}+\text { the } \Lambda_{n}^{1} \text {-comprehension scheme. }
$$

The following lemma is easily proved by syntactic manipulations of formulas in $\Lambda_{n}^{1}$.

Lemma 1.3. If $n<\omega$ and $(\mathscr{N}, \mathscr{X}) \vDash \Pi_{n+1}^{1}-\mathrm{CA}_{0}+\sum_{n}^{1}$-Coll, then $(\mathscr{N}, \mathscr{X}) \vDash$ $\Lambda_{n+1}^{1}-\mathrm{CA}_{0}$.

The following well-known theorem is proved by constructing the ramified analaytical hierarchy inside the model $(\mathscr{N}, \mathscr{X})$.

TheOREM 1.4. Suppose $n<\omega$ and $(\mathscr{N}, \mathscr{X}) \vDash \Pi_{n}^{1}-\mathrm{CA}_{0}$. Then there is $\mathscr{X}^{\prime} \subseteq \mathscr{X}$ such that $\left(\mathscr{N}, \mathscr{X}^{\prime}\right) \vDash \Pi_{n}^{1}-\mathrm{CA}_{0}+\Sigma_{n}^{1}-\mathrm{AC}_{0}$.

By a modification of the proof of Theorem 1.4 we will prove the following theorem which will be applied in $\S 4$.

TheOREM 1.5. Suppose $n<\omega$ and $(\mathscr{N}, \mathscr{X}) \vDash \Lambda_{n}^{1}-\mathrm{CA}_{0}$. Then there is $\mathscr{X}^{\prime} \subseteq \mathscr{X}$ such that $\left(\mathscr{N}, \mathscr{X}^{\prime}\right) \vDash \Pi_{n}^{1}-\mathrm{CA}_{0}+\sum_{n}^{1}-\mathrm{AC}_{0}$.

Proof. In the case that $n=0$ just let $\mathscr{X}^{\prime}=\mathscr{X}$. If $n=1$, then let $\mathscr{X}^{\prime}$ be the set of those $X \in \mathscr{X}$ which are $\Lambda_{n}^{1}$-definable. Then $\left(\mathscr{N}, \mathscr{X}^{\prime}\right)$ is a (reduced) model of $\Pi_{1}^{1}-\mathrm{CA}_{0}$ (see [8]), and thus also a model of $\Sigma_{1}^{1}-\mathrm{AC}_{0}$. Assume from now on that $n \geqslant 2$.

We begin by sketching the development of the ramified analytical hierarchy in a way suitable for our purpose. The following definitions are made in the structure $(\mathscr{N}, \mathscr{X})$.

A well-ordered set $W$ is a pair $\langle D, R\rangle$, where $R$ well-orders $D$. We set $\mathscr{D}(W)=D$ and write $x<_{W} y$ in case $\langle x, y\rangle \in R$. If $b \in \mathscr{D}(W)$, then $W \mid b=\langle\mathscr{D}(W \mid b)$, $R \cap(\mathscr{D}(W \mid b) \times \mathscr{D}(W \mid b))\rangle$, where $\mathscr{D}(W \mid b)=\left\{x \in \mathscr{D}(W): x<_{W} b\right\}$. The terms $W$-minimal, $W$-successor, $W$-limit point, etc., have their obvious meanings. If $W$ and $V$ are well-ordered sets, then we write $W \leqq V$ when $W$ is isomorphic to an initial segment of $V$, and $W \approx V$ when both $W \leqslant V$ and $V \leqslant W$. If either $W \leqslant V$ or $V \leqslant W$, then $W$ and $V$ are comparable. Note that it is possible for there to be incomparable well-ordered sets. 
Let $\Phi(x, y, z, Z)$ be an arithmetic formula (with all of its free variables displayed) having the following two properties:

(1) If $\phi(X, x)$ is a $\Pi_{n}^{1}$ formula (which may have first-order parameters from $N$ ), and if $A \in \mathscr{X}$ and $a, b \in N$, then

$$
\left.\left(\mathscr{N},\left\{(A)_{i}: i \in N\right\}\right) \vDash \phi\left((A)_{b}, a\right) \quad \text { iff } \quad(\mathscr{N}, \mathscr{X}) \vDash \Phi\left(a,{ }^{\ulcorner} \phi\right\urcorner, b, A\right) .
$$

(2) Suppose $A, B \in \mathscr{X}$ and $a, b \in N$ are such that $\left\{(A)_{i}: i \in N\right\}=\left\{(B)_{i}\right.$ : $i \in N\}$ and $(A)_{a}=(B)_{b}$. Then

$$
(\mathscr{N}, A, B) \vDash \forall x y[\Phi(x, y, a, A) \leftrightarrow \Phi(x, y, b, B)] .
$$

Then define the operator $j$ by

$$
j(Z)=\{\langle\langle y, z\rangle, x\rangle: \Phi(x, y, z, Z)\} .
$$

This operator is well defined since $(\mathscr{N}, \mathscr{X}) \vDash \mathrm{ACA}_{0}$.

Next we will iterate the operator $j$ along a well-ordered set $W$ to obtain $\varnothing^{(W)}$. If $\mathscr{D}(W)=\varnothing$, then $\varnothing^{(W)}=\varnothing$; so assume $\mathscr{D}(W) \neq \varnothing$. For $a \in \mathscr{D}(W)$ inductively define $J(a)$ as follows:

$$
J(a)=\left\{\begin{array}{l}
\varnothing \quad \text { if } a \text { is } W \text {-minimal; } \\
j(J(b)) \text { if } a \text { is the } W \text {-successor of } b \\
\left\{\langle\langle x, y\rangle, z\rangle: x<_{W} a \text { and }\langle y, z\rangle \in J(x)\right\} \quad \text { if } a \text { is a } W \text {-limit point. }
\end{array}\right.
$$

If $W$ has a maximal element $a$, then set $\varnothing^{(W)}=j(J(a))$; otherwise let $\varnothing^{(W)}=$ $\{\langle\langle x, y\rangle, z\rangle: x \in \mathscr{D}(W)$ and $\langle y, z\rangle \in J(x)\}$.

It is not necessary that $\varnothing^{(W)}$ exist; however, if it does, then it is unique. This leads us to the notion of a proper well-ordered set.

A well-ordered set $W$ is proper provided that the following two conditions are satisfied:

$$
\begin{aligned}
& \varnothing^{(W)} \text { exists; for each } a \in \mathscr{D}(W) \text { there is some } b \in N \text { such that } W \mid a \approx \\
& \left(j\left(\varnothing^{(W \mid a)}\right)\right)_{b} .
\end{aligned}
$$

If $W$ is a proper well-ordered set, then let $\mathscr{R} \mathscr{A}(W)=\left\{\left(\varnothing^{(W)}\right)_{x}: x \in N\right\}$. If $V$ and $W$ are proper well-ordered sets, then $V \leqslant W$ iff $\mathscr{R} \mathscr{A}(V) \subseteq \mathscr{R} \mathscr{A}(W)$. Also, notice that any two proper well-ordered sets are comparable.

We now define $\mathscr{R} \mathscr{A}$, the collection of sets in the ramified analytical hierarchy, as

$$
\bigcup\{\mathscr{R} \mathscr{A}(W): W \text { is a proper well-ordered set }\} \text {. }
$$

The collection $\mathscr{R} \mathscr{A}$ is definable by a $\Sigma_{2}^{1}$ formula.

If $\mathscr{Y} \subseteq \mathscr{X}$, then we will identify $\mathscr{Y}$ with the structure $(\mathscr{N}, \mathscr{Y})$. If $\mathscr{Y}_{1} \subseteq \mathscr{Y}_{2} \subseteq \mathscr{X}$ and $m<\omega$, then we will write $\mathscr{Y}_{1} \prec_{m} \mathscr{Y}_{2}$ if whenever $\sigma$ is a $\Sigma_{m}^{1}$ sentence with parameters from $N$ and $\mathscr{Y}_{1}$, then $\mathscr{Y}_{1} \vDash \sigma$ iff $\mathscr{Y}_{2} \vDash \sigma$.

There is a $\Delta_{2}^{1}$ "well-ordering" of $\mathscr{R} \mathscr{A}$ which is absolute in the following sense. Let us say that $\mathscr{I} \subseteq \mathscr{R} \mathscr{A}$ is an initial segment of $\mathscr{R} \mathscr{A}$ if whenever $W$ is a proper well-ordered set and $\mathscr{I} \cap \mathscr{R} \mathscr{A}(W) \neq \varnothing$, then $\mathscr{I} \supseteq \mathscr{R} \mathscr{A}(W)$. In particular, if $W$ is a proper well-ordered set, then $\mathscr{R} \mathscr{A}(W)$ is an initial segment. Now let $\theta(X, Y)$ be the $\Delta_{2}^{1}$ "formula" defining < $<_{\mathscr{R} A}$. Then for any initial segment $\mathscr{I}$ with $A, B \in \mathscr{I}$,

$$
\mathscr{X} \vDash \theta(A, B) \text { iff } \mathscr{I} \vDash \theta(A, B) .
$$


If $W \leqq V$ are proper well-ordered sets, then $\mathscr{R} \mathscr{A}(V)$ is an end-extension of $\mathscr{R} \mathscr{A}(W)$ in that whenever $A \in \mathscr{R} \mathscr{A}(W)$ and $B \in \mathscr{R} \mathscr{A}(V)-\mathscr{R} \mathscr{A}(W)$, then $A<\mathscr{R} \mathscr{A}$ $B$. It will then follow that if $\mathscr{I}$ is an initial segment of $\mathscr{R} \mathscr{A}, i \leqslant n$, and $\mathscr{I} \vDash \Pi_{i}^{1}-\mathrm{CA}_{0}$, then $\mathscr{I} \vDash \Sigma_{i}^{1}-\mathrm{AC}_{0}$.

The "well-orderedness" of $<\mathscr{R} A$ permits us to define ordinals. An ordinal is a proper well-ordered set $W$ in $\mathscr{R} \mathscr{A}$ such that whenever $V$ is a well-ordered set in $\mathscr{R} \mathscr{A}$ such that $W \approx V$, then $W \leqslant \mathscr{R} \& V$. We will say that a well-ordered set $W$ has an ordinal if $W \approx V$ for some ordinal $V$. It can be proved by induction on proper well-ordered sets that every proper well-ordered set has a unique ordinal.

We now present the definition of $<_{\mathscr{R} A}$. If $X, Y \in \mathscr{R} \mathscr{A}$, then $X<_{\mathscr{R} A} Y$ iff one of the following three conditions holds:

(1) There is a proper well-ordered set $W$ such that $X \in \mathscr{R} \mathscr{A}(W)$ and $Y \notin \mathscr{R} \mathscr{A}(W)$.

(2) There is a proper well-ordered set $W_{0}$ such that $X, Y \notin \mathscr{R} \mathscr{A}\left(W_{0}\right)$ yet for any well-ordered set $W>W_{0}, X, Y \in \mathscr{R} \mathscr{A}(W)$. Furthermore, the least $y_{1}$ such that $X=\left\{x \in N:(\mathscr{N}, \mathscr{X}) \vDash \Phi\left(x, y_{1}, z_{1}, \varnothing^{\left(W_{0}\right)}\right)\right\}$ for some $z_{1}$ is less than the least $y_{2}$ such that $Y=\left\{x \in N:(\mathscr{N}, \mathscr{X}) \vDash \Phi\left(x, y_{2}, z_{2}, \varnothing^{\left(W_{0}\right)}\right)\right\}$ for some $z_{2}$.

(3) There is $W_{0}$ as in (2), and for $y_{1}$ and $y_{2}$ defined in (2), $y_{1}=y_{2}$. Let $\ulcorner\phi(X, x)\urcorner=y_{1}$. Then the $<\mathscr{R} \mathscr{A}$ - least $B_{1} \in \mathscr{R} \mathscr{A}\left(W_{0}\right)$ such that $\left\{x \in N: \mathscr{R} \mathscr{A}\left(W_{0}\right) \vDash\right.$ $\left.\phi\left(B_{1}, x\right)\right\}$ is $<_{\mathscr{A} \mathscr{A}}$ the $<_{\mathscr{R} \mathscr{A}}$-least $B_{2} \in \mathscr{R} \mathscr{A}\left(W_{0}\right)$ such that $Y=\left\{x \in N: \mathscr{R} \mathscr{A}\left(W_{0}\right)\right.$ $\left.\vDash \phi\left(B_{2}, x\right)\right\}$.

We will first investigate what happens if there is a $\Lambda_{n}^{1}$-definable well-ordered set having no ordinal.

LEMMA 1.5.1. Suppose there is a $\Lambda_{n}^{1}$-definable well-ordered set having no ordinal. Then $\mathscr{R} \mathscr{A} \vDash \Pi_{n}^{1}-\mathrm{CA}_{0}$.

Proof. Let $W_{0}$ be a minimal $\Lambda_{n}^{1}$-definable well-ordered set having no ordinal. Such a set exists, and $\mathscr{R} \mathscr{A}=\mathscr{R} \mathscr{A}\left(W_{0}\right)$. Clearly, $\mathscr{R} \mathscr{A}\left(W_{0}\right) \vDash \Pi_{0}^{1}-\mathrm{CA}_{0}$. We will prove by induction on $i<n$ that $\mathscr{R} \mathscr{A}\left(W_{0}\right) \vDash \Pi_{i+1}^{1}-\mathrm{CA}_{0}$, so suppose that $\mathscr{R} \mathscr{A}\left(W_{0}\right) \vDash \Pi_{i}^{1}$ $\mathrm{CA}_{0}$.

Let $A \in \mathscr{R} \mathscr{A}\left(W_{0}\right)$ and let $\phi(x, Y, A)$ be a $\Pi_{i}^{1}$ formula. We claim there is an ordinal $W \in \mathscr{R} \mathscr{A}\left(W_{0}\right)$ such that

$$
\mathscr{R} \mathscr{A}\left(W_{0}\right) \vDash \forall x[\exists Y \phi(x, Y, A) \rightarrow \exists Y \in \mathscr{R} \mathscr{A}(W) \phi(x, Y, A)] .
$$

For, if not, let $\psi(x, A, W)$ be the formula

$$
\begin{aligned}
\text { “W is an ordinal" } & \wedge \exists Y \in \mathscr{R} \mathscr{A}(W) \phi(x, Y, A) \\
& \wedge \forall w \in \mathscr{D}(W)_{\neg} \exists Y \in \mathscr{R} \mathscr{A}(W \mid w) \phi(x, Y, A) .
\end{aligned}
$$

Now define $D_{0}$ and $R_{0}$ by

$$
\langle x, w\rangle \in D_{0} \leftrightarrow \mathscr{R} \mathscr{A}\left(W_{0}\right) \vDash \exists W(\psi(x, A, W) \wedge w \in \mathscr{D}(W))
$$

and

$$
\begin{array}{r}
\langle x, w\rangle R_{0}\left\langle x^{\prime}, w^{\prime}\right\rangle \leftrightarrow \mathscr{R} \mathscr{A}\left(W_{0}\right) \vDash \exists W\left[\psi(x, A, W) \wedge\langle x, w\rangle \in D_{0} \wedge\left\langle x^{\prime}, w^{\prime}\right\rangle \in D_{0}\right. \\
\left.\wedge\left(x<x^{\prime} \vee\left(x=x^{\prime} \wedge w<{ }_{w} w^{\prime}\right)\right)\right] .
\end{array}
$$


These definitions are $\Lambda_{n}^{1}$, so the well-ordered set $V_{0}=\left\langle D_{0}, R_{0}\right\rangle$ exists and is $\Lambda_{n}^{1}$-definable. Clearly, $W_{0} \lesssim V_{0}$ and $V_{0} \in j\left(\phi^{\left(W_{0}\right)}\right)$. This contradicts the defining property of $W_{0}$, so there exists $W \in \mathscr{R} \mathscr{A}\left(W_{0}\right)$ satisfying $(*)$.

By the inductive hypothesis, there is $B \in \mathscr{R} \mathscr{A}\left(W_{0}\right)$ such that

$$
\mathscr{R} \mathscr{A}\left(W_{0}\right) \vDash \forall x(x \in B \leftrightarrow \exists Y \in \mathscr{R} \mathscr{A}(W) \phi(x, Y, A)),
$$

and then for this same set $B$,

$$
\mathscr{R} \mathscr{A}\left(W_{0}\right) \vDash \forall x(x \in B \leftrightarrow \exists Y \phi(x, Y, A)) .
$$

We will assume for the remainder of this proof that every $\Lambda_{n}^{1}$-definable well-ordered set has an ordinal.

Lemma 1.5.2. Suppose $V$ is a $\Lambda_{n}^{1}$-definable well-ordered set. Then there is a least ordinal $W \in \mathscr{R} \mathscr{A}$ such that $\mathscr{R} \mathscr{A}(V) \subseteq \mathscr{R} \mathscr{A}(W) \prec{ }_{1} \mathscr{X}$.

Proof. The proof of the lemma is in several steps which we now sketch.

The first step is to prove: If $\phi(V, Y)$ is arithmetic and $\mathscr{X} \vDash \exists Y \phi(V, Y)$, then $\mathscr{R} \mathscr{A} \vDash \exists Y \phi(V, Y)$. Assume $\mathscr{X} \vDash \exists Y \phi(V, Y)$. By the Kleene Normal Form Theorem (which is provable in $\mathrm{ACA}_{0}$ and hence holds in both $\mathscr{X}$ and $\mathscr{R} \mathscr{A}$ (see [8])) there is a tree $T$ which is arithmetic in $V$ such that its set of branches is recursively isomorphic to the set of witnesses of $\phi(V, Y)$. Since $\mathscr{X} \vDash \exists Y \phi(V, Y)$, then $\mathscr{X} \vDash$ " $T$ is not well-founded". There is a well-ordered set $H$ (unique up to $\approx$ ), a subset $T_{0} \subseteq T$, and a function $R: T_{0} \rightarrow \mathscr{D}(H)$ such that $R$ is onto, for each $x \in T_{0} R(x)=a$ iff $x$ is a minimal element of the subset $\left\{t \in T: R(t)<{ }_{H} a\right\}$, and $T-T_{0}$ has no minimal elements. Then $H$ is $\Lambda_{n}^{1}$-definable, hence is in $\mathscr{R} \mathscr{A}$. Clearly, then $T-T_{0}$ is also in $\mathscr{R} \mathscr{A}$, and so a branch of $T$ is also in $\mathscr{R} \mathscr{A}$.

The second step is to prove: If $\phi(x, Y, V)$ is arithmetic, then there is an ordinal $W \in \mathscr{R} \mathscr{A}$ such that

$$
\begin{aligned}
\mathscr{R} \mathscr{A} \vDash & \forall x(\exists Y \phi(x, Y, V) \rightarrow \exists Y \in \mathscr{R} \mathscr{A}(W) \phi(x, Y, V)) \\
& \wedge \forall y \in \mathscr{D}(W) \exists x(\exists Y \phi(x, Y, V) \neg \exists Y \in \mathscr{R} \mathscr{A}(W \mid y) \phi(x, Y, V)) .
\end{aligned}
$$

The proof of this is just like the proof of Lemma 1.5.1.

The third step is to complete the proof of the lemma. Let $\phi(X, Y)$ be a complete $\Pi_{1}^{0}$ formula. We will show that there is a least ordinal $W \in \mathscr{R} \mathscr{A}$ such that $\mathscr{R} \mathscr{A}(V) \subseteq \mathscr{R} \mathscr{A}(W)$ and

$$
\mathscr{R} \mathscr{A} \vDash \forall X \in \mathscr{R} \mathscr{A}(W)[\exists Y \phi(X, Y) \rightarrow \exists Y \in \mathscr{R} \mathscr{A}(W) \phi(X, Y)] .
$$

We can suppose $V \in \mathscr{R} \mathscr{A}$, and that $W=V$ does not work. Let us suppose there is no such ordinal $W$. Consider the formula $\psi(u, V, Z)$ :

$$
\begin{aligned}
& \forall z \leqslant u\left((Z)_{z} \text { is an ordinal }\right) \\
& \wedge V \in \mathscr{R} \mathscr{A}\left((Z)_{0}\right) \wedge \forall z \in \mathscr{D}\left((Z)_{0}\right) V \notin \mathscr{R} \mathscr{A}\left((Z)_{0} \mid z\right) \\
& \wedge \forall z, w\left(z<w \leqslant u \rightarrow \forall X \in \mathscr{R} \mathscr{A}\left((Z)_{z}\right)\right. \\
& \times\left(\exists Y \phi(X, Y) \rightarrow \exists Y \in \mathscr{R} \mathscr{A}\left((Z)_{w}\right) \phi(X, Y)\right) \\
& \wedge \forall y \in \mathscr{D}\left((Z)_{w}\right) \exists X \in \mathscr{R} \mathscr{A}\left((Z)_{z}\right) \\
& \left.\quad \times\left(\exists Y \phi(X, Y) \wedge \neg \exists Y \in \mathscr{R} \mathscr{A}\left((Z)_{w} \mid y\right) \phi(X, Y)\right)\right) .
\end{aligned}
$$


Define a set $J$ by $u \in J \leftrightarrow \exists Z \psi(u, V, Z)$. The set $J$ exists since it is $\Lambda_{n}^{1}$-definable, so by the second step of this proof, $J=N$. Now define $D_{1}$ and $R_{1}$ by

$$
\langle u, w\rangle \in D_{1} \leftrightarrow \exists Z\left[\psi(u, V, Z) \wedge w \in \mathscr{D}\left((Z)_{u}\right)\right]
$$

and

$$
\begin{aligned}
\langle u, w\rangle R_{1}\left\langle u^{\prime}, w^{\prime}\right\rangle \leftrightarrow & \langle u, w\rangle \in D_{1} \wedge\left\langle u^{\prime}, w^{\prime}\right\rangle \in D_{1} \\
& \wedge\left[u<u^{\prime} \vee\left(u=u^{\prime} \wedge \exists Z \psi\left((u, V, Z) \wedge w<_{(Z)_{u}} w^{\prime}\right)\right)\right] .
\end{aligned}
$$

Then $W_{1}=\left\langle D_{1}, R_{1}\right\rangle$ is a well-ordered set which is $\Lambda_{n}^{1}$-definable. Thus, $W_{1}$ has an ordinal $W \in \mathscr{R} \mathscr{A}$, and $W$ has the desired properties.

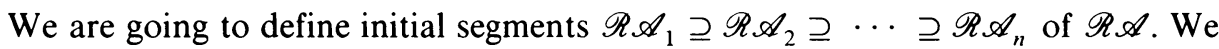
define $\mathscr{R} \mathscr{A}_{1}$, where $\mathscr{R} \mathscr{A}_{1}=\bigcup\left\{\mathscr{R} \mathscr{A}(W): \mathscr{R} \mathscr{A}(W) \prec_{1} \mathscr{X}\right\}$. If $W$ is an ordinal such that $\mathscr{R} \mathscr{A}(W) \prec{ }_{1} \mathscr{X}$, then $\mathscr{R} \mathscr{A}(W) \prec{ }_{1} \mathscr{R} \mathscr{A}_{1}$. By Lemma 1.5 .2 there is an ordinal $W$ such that $\mathscr{R} \mathscr{A}(W) \prec{ }_{1} \mathscr{X}$. Furthermore, there is no largest such ordinal, for if there were it would have to be $\Lambda_{n}^{1}$-definable. Therefore, the following statement (1.i) for $i=1$ is true:

(1.i) There are arbitrarily large ordinals $W \in \mathscr{R} \mathscr{A}_{i}$ such that $\mathscr{R} \mathscr{A}(W) \prec_{i} \mathscr{R} \mathscr{A}_{i}$.

For $1 \leqslant i \leqslant n-2$, we define

$$
\mathscr{R} \mathscr{A}_{i+1}=\bigcup\left\{\mathscr{R} \mathscr{A}(W): \mathscr{R} \mathscr{A}(W) \prec_{i+1} \mathscr{R} \mathscr{A}_{i}\right\}
$$

and we define

$$
\mathscr{R} \mathscr{A}_{n}=\bigcup\left\{\mathscr{R} \mathscr{A}\left(W_{k}\right): k<\omega\right\},
$$

where $W_{k}$ is the $k$ th ordinal in $\mathscr{R} \mathscr{A}_{n-1}$ such that $\mathscr{R} \mathscr{A}\left(W_{k}\right) \prec_{n} \mathscr{R} \mathscr{A}_{n-1}$.

Consider the following two statements:

(2.i) $\mathscr{R} \mathscr{A}_{i} \vDash \Pi_{i}^{1}-\mathrm{CA}_{0}$.

(3.i) If $V$ is a $\Lambda_{n}^{1}$-definable well-ordered set, then there is a least ordinal $W \in \mathscr{R} \mathscr{A}_{i}$ such that $\mathscr{R} \mathscr{A}(V) \subseteq \mathscr{R} \mathscr{A}(W) \prec_{i+1} \mathscr{R} \mathscr{A}_{i}$.

Our goal is to prove $(2 . n)$. Our strategy for doing this is to prove the chain of implications

$$
(1.1) \Rightarrow(2.1) \Rightarrow(3.1) \Rightarrow(1.2) \Rightarrow \cdots \Rightarrow(1 . n) \Rightarrow(2 . n) .
$$

LEMMA 1.5.3. For $1 \leqslant i \leqslant n,(1 . i) \Rightarrow(2 . i)$.

Proof. Consider some $\Pi_{i}^{1}$ formula $\phi(x, A)$, where $A \in \mathscr{R} \mathscr{A}_{i}$. Let $W \in \mathscr{R} \mathscr{A}_{i}$ be an ordinal such that $A \in \mathscr{R} \mathscr{A}(W) \prec{ }_{i} \mathscr{R} \mathscr{A}_{i}$.

The formula " $\mathscr{R} \mathscr{A}(W) \vDash \phi(x, A)$ " is arithmetic in the parameters $\varnothing^{(W)}$ and $A$, so that there is $B \in \mathscr{R} \mathscr{A}_{i}$ such that for each $b \in N, b \in B \Leftrightarrow \mathscr{R} \mathscr{A}(W) \vDash \phi(b, A)$. But then $b \in B \Leftrightarrow \mathscr{R} \mathscr{A}_{i} \vDash \phi(b, A)$, so that $\mathscr{R} \mathscr{A}_{i} \vDash \exists X \forall x(x \in X \leftrightarrow \phi(x, A))$.

LEMMA 1.5.4. For $1 \leqslant i<n,(2 . i) \Rightarrow(3 . i)$.

The proof of this lemma is much like the proof of Lemma 1.5.2, and we omit it.

LeMma 1.5.5. For $1 \leqslant i<n,(3 . i) \Rightarrow(1 . i+1)$.

Proof. There are two cases to consider, depending on whether $i \leqslant n-2$ or $i=n-1$. 
First, suppose $i \leqslant n-2$. We just need to show that there is no largest ordinal $W \in \mathscr{R} \mathscr{A}_{i}$ such that $\mathscr{R} \mathscr{A}(W) \prec_{i+1} \mathscr{R} \mathscr{A}_{i}$. By noticing that $\mathscr{R} \mathscr{A}_{i}$ is definable by a $\Sigma_{i+1}^{1}$ formula, we can check that if $W$ is such a largest ordinal, then $W$ is $\Lambda_{i+2}^{1}$-definable. This contradicts (3.i).

Next, suppose $i=n-1$. Again, using that $\mathscr{R} \mathscr{A}_{n-1}$ is definable by a $\Sigma_{n}^{1}$ formula we can check by induction on $k<\omega$ that $W_{k}$, the $k$ th ordinal $W$ such that $\mathscr{R} \mathscr{A}(W) \prec_{n} \mathscr{R} \mathscr{A}_{n-1}$, is $\Lambda_{n}^{1}$-definable, so that the ordinal $W_{k+1}$ exists.

2. Natural models. This section is concerned exclusively with some model theory of the familiar subtheories of true second-order arithmetic. When considering an extension $\left(\mathscr{N}^{\prime}, \mathscr{X}^{\prime}\right)$ of a model $(\mathscr{N}, \mathscr{X})$ of some second-order theory, we leave as tacitly understood the existence of an injection $e: \mathscr{X} \rightarrow \mathscr{X}^{\prime}$. We will conventionally let $A^{\prime}=e(A)$ for each $A \in \mathscr{X}$, and if $\phi$ is a formula in which parameters from $(\mathscr{N}, \mathscr{X})$ occur, then $\phi^{\prime}$ will be the formula obtained from $\phi$ by replacing each parameter $A \in \mathscr{X}$ by $A^{\prime}$.

Definition 2.1. Suppose $n<\omega$ and $\left(\mathscr{N}^{\prime}, \mathscr{X}^{\prime}\right)$ is an extension of $(\mathscr{N}, \mathscr{X})$. Then $\left(\mathscr{N}^{\prime}, \mathscr{X}^{\prime}\right)$ is a $\Sigma_{n}^{1}$-extension of $(\mathscr{N}, \mathscr{X})$ (in symbols: $(\mathscr{N}, \mathscr{X}) \prec_{n}\left(\mathscr{N}^{\prime}, \mathscr{X}^{\prime}\right)$ ) if, for every $\Sigma_{n}^{1}$-sentence $\phi$ in which parameters from both $\mathscr{N}$ and $\mathscr{X}$ are permitted,

$$
(\mathscr{N}, \mathscr{X}) \vDash \phi \quad \text { iff } \quad\left(\mathscr{N}^{\prime}, \mathscr{X}^{\prime}\right) \vDash \phi^{\prime} .
$$

Definition 2.2. If $(\mathscr{N}, \mathscr{X}) \prec_{0}\left(\mathscr{N}^{\prime}, \mathscr{X}^{\prime}\right)$, then the extension is exclusive if for each $B \in \mathscr{X}^{\prime}$ there are $A \in \mathscr{X}$ and $a \in \mathscr{N}^{\prime}$ such that $\left(\mathscr{N}^{\prime}, \mathscr{X}^{\prime}\right) \vDash B=\left(A^{\prime}\right)_{a}$.

Let $\mathscr{L}$ be an unspecified countable language extending the language of PA. We let PA* be the first-order $\mathscr{L}$-theory which extends PA and contains all instances of the induction axioms for formulas in $\mathscr{L}$. Now suppose $\mathscr{N} \vDash \operatorname{PA}^{*}$. We let $\operatorname{Def}(\mathscr{N})$ be the set of all parametrically definable subsets of $\mathscr{N}$. A subset $X \subseteq N$ is a class if for each $a \in N,\{x \in X: x \leqslant a\} \in \operatorname{Def}(\mathscr{N})$. We let Class $(\mathscr{N})$ denote the set of all classes of $\mathscr{N}$.

Rather classless models $\mathscr{N}$ of $\mathrm{PA}^{*}$ have played an important role in the (first-order) model theory of models of PA*. Recall (from [1 and 7]) that $\mathscr{N}$ is rather classless if $\operatorname{Class}(\mathscr{N})=\operatorname{Def}(\mathscr{N})$. The next definition is a sort of extension of this notion to models of $\mathrm{ACA}_{0}$.

Definition 2.3. Let $(\mathscr{N}, \mathscr{X}) \vDash \operatorname{ACA}_{0}$. Then $(\mathscr{N}, \mathscr{X})$ is natural iff $\mathscr{X}=\operatorname{Class}(\mathscr{N})$.

In particular, notice that $\mathscr{N}$ is rather classless iff $(\mathscr{N}, \operatorname{Def}(\mathscr{N}))$ is natural. Any nonstandard natural model has uncountable cofinality. More generally, we have the following necessary and sufficient condition for $\mathscr{N}$ to be natural. For any model $\mathscr{N}$ and $X \in \operatorname{Class}(\mathcal{N})$, we say that the length of $X$ is the unique initial segment $I \subseteq N$ (which may be equal to $N$ ) such that for some $F \in \operatorname{Class}(\mathscr{N}),\left\{\left\langle(f)_{0}:(f)_{1}\right\rangle\right.$ : $f \in F\}$ is an order-preserving bijection from $X$ onto $I$. We say that $X$ is long provided its length is $N$.

Proposition 2.4. $(\mathscr{N}, \operatorname{Class}(\mathscr{N})) \vDash \mathrm{ACA}_{0}$ iff every unbounded class of $\mathscr{N}$ is long.

Proof. Suppose $(\mathscr{N}, \operatorname{Class}(\mathscr{N})) \vDash \operatorname{ACA}_{0}$, and let $X \in \operatorname{Class}(\mathscr{N})$ be unbounded. Then the length of $X$ is definable in $(\mathscr{N}, X)$ and, having no maximal element, must be $N$. 
Conversely, suppose every unbounded class is long. We need only show that for every $A \in \operatorname{Class}(\mathscr{N})$ and every $\Delta_{0}$ formula $\Theta(A, x, y)$, that $X=\{a \in N: \mathscr{N} \vDash$ $\exists y \theta(A, a, y)\}$ is a class. Suppose $X$ is not a class. Then there is $b \in N$ such that $B=\{a \in X: a \leqslant b\}$ is not a class. For each $c \in N$ let $B_{c}=\{a \in B: \mathscr{N} \vDash \exists y \leqslant$ $c \theta(A, a, y)\}$. Let $C=\left\{c \in N: B_{c} \neq B_{c+1}\right\}$. Then $C$ is a class since for each $d \in C$ there is $m \in N$ such that if $A_{m}=\{x \in A: x \leqslant m\}$, then

$$
\mathscr{N} \vDash \forall x \forall z \leqslant d\left[\exists y \leqslant z \theta(A, x, y) \leftrightarrow \exists y \leqslant z \theta\left(A_{m}, x, y\right)\right] .
$$

Also, by considering the function $F: C \rightarrow N$ where $F(c)=\min \left(B_{c+1}-B_{c}\right)$, we see that the length of $C$ is a subset of $B$, a contradiction.

We say that a linearly ordered set $(A,<)$ has strict cofinality $\kappa$ if it has cofinality $\kappa$ and it has no subset of cardinality $\kappa$ which either is well-ordered and bounded or is inversely well-ordered. If for some regular $\kappa,(A,<)$ has strict cofinality $\kappa$, then we say that $(A,<)$ has strict cofinality. Notice that any $(A,<)$ which is $\kappa$-like for some regular $\kappa$ has strict cofinality. Also notice that if a model $\mathscr{N}$ has strict cofinality, then every unbounded class is long, so that by Proposition $2.4,(\mathscr{N}$, Class $(\mathscr{N})) \vDash$ $\mathrm{ACA}_{0}$.

Nonstandard natural models of $\Pi_{1}^{1}-\mathrm{CA}_{0}$ were constructed in [8]; it was asked there if nonstandard natural models of larger fragments of true second-order arithmetic, such as $\Delta_{2}^{1}-C_{0}$ or even $\mathrm{CA}+\mathrm{AC}$, exist. These questions have affirmative answers; the next theorem is the key to constructing such models.

THEOREM 2.5. Suppose that $n<\omega,(\mathscr{N}, \mathscr{X}) \prec_{0}\left(\mathscr{N}^{\prime}, \mathscr{X}^{\prime}\right)$ exclusively, and $(\mathscr{N}, \mathscr{X})$ $\vDash \sum_{n}^{1}$-Coll. Then $(\mathscr{N}, \mathscr{X}) \prec_{n+1}\left(\mathscr{N}^{\prime}, \mathscr{X}^{\prime}\right)$.

Proof. First we show that $(\mathscr{N}, \mathscr{X}) \prec_{1}\left(\mathscr{N}^{\prime}, \mathscr{X}^{\prime}\right)$. Suppose $\left(\mathscr{N}^{\prime}, \mathscr{X}^{\prime}\right) \vDash \exists X \phi^{\prime}(X)$, where $\phi(X)$ is a $\Pi_{0}^{1}$-formula, possibly involving some parameters from $(\mathscr{N}, \mathscr{X})$. Then there are $A \in \mathscr{X}$ and $a \in N^{\prime}$ such that $\left(\mathscr{N}^{\prime}, \mathscr{X}^{\prime}\right) \vDash \phi^{\prime}\left(\left(A^{\prime}\right)_{a}\right)$. Thus, $\left(\mathscr{N}^{\prime}, \mathscr{X}^{\prime}\right)$ $\vDash \exists x \phi^{\prime}\left(\left(A^{\prime}\right)_{x}\right)$, so that $(\mathscr{N}, \mathscr{X}) \vDash \exists x \phi\left((A)_{x}\right)$. But then $(\mathscr{N}, \mathscr{X}) \vDash \exists X \phi(X)$.

We now proceed by induction on $n$, so we assume that $n \geqslant 1$ and $(\mathscr{N}, \mathscr{X}) \prec_{n}$ $\left(\mathscr{N}^{\prime}, \mathscr{X}^{\prime}\right)$. Suppose $\left(\mathscr{N}^{\prime}, \mathscr{X}^{\prime}\right) \vDash \exists X \forall Y \phi^{\prime}(X, Y)$, where $\phi(X, Y)$ is $\Sigma_{n-1}^{1}$. Then there is $A \in \mathscr{X}$ such that

$$
\left(\mathscr{N}^{\prime}, \mathscr{X}^{\prime}\right) \vDash \exists x \forall Y \phi^{\prime}\left(\left(A^{\prime}\right)_{x}, Y\right) .
$$

We claim that $(\mathscr{N}, \mathscr{X}) \vDash \exists x \forall Y \phi\left((A)_{x}, Y\right)$. If not, then $(\mathscr{N}, \mathscr{X}) \vDash$ $\forall x \exists Y \neg \phi\left((A)_{x}, Y\right)$, so that by $\Sigma_{n}^{1}$-Coll, $(\mathscr{N}, \mathscr{X}) \vDash \exists Y \forall x \exists y \neg \phi\left((A)_{x},(Y)_{y}\right)$. Thus, there is $B \in \mathscr{X}$ such that $(\mathscr{N}, \mathscr{X}) \vDash \forall x \exists y \neg \phi\left((A)_{x},(B)_{y}\right)$. Since $\Sigma_{n}^{1}$-Coll implies $\Pi_{n-1}^{1}-\mathrm{CA}_{0}$ (Lemma 1.1) there is $C \in \mathscr{X}$ such that $(\mathscr{N}, \mathscr{X}) \vDash \forall x y(\langle x, y\rangle \in C \leftrightarrow$ $\left.\neg \phi\left((A)_{x},(B)_{y}\right)\right)$. This last sentence is $\Pi_{n}^{1}$ so that by the inductive hypothesis,

$$
\left(\mathscr{N}^{\prime}, \mathscr{X}^{\prime}\right) \vDash \forall x y\left(\langle x, y\rangle \in C^{\prime} \leftrightarrow \neg \phi^{\prime}\left(\left(A^{\prime}\right)_{x},\left(B^{\prime}\right)_{y}\right)\right) .
$$

Also, $\left(\mathscr{N}^{\prime}, \mathscr{X}^{\prime}\right) \vDash \forall x \exists y\left(\langle x, y\rangle \in C^{\prime}\right)$. Therefore, $\left(\mathscr{N}^{\prime}, \mathscr{X}^{\prime}\right) \vDash$ $\forall x \exists y \neg \phi^{\prime}\left(\left(A^{\prime}\right)_{x},\left(B^{\prime}\right)_{y}\right)$, and this implies $\left(\mathscr{N}^{\prime}, \mathscr{X}^{\prime}\right) \vDash \forall x \exists Y \neg \phi^{\prime}\left(\left(A^{\prime}\right)_{x}, Y\right)$, contradicting (*). This proves the claim, so that, in particular, $(\mathscr{N}, \mathscr{X}) \vDash \exists X \forall Y \phi(X, Y)$. 
The previous theorem has a couple of interesting refinements, which are the following two theorems.

THEOREM 2.6. Suppose that $n<\omega,(\mathscr{N}, \mathscr{X}) \prec_{0}\left(\mathscr{N}^{\prime}, \mathscr{X}^{\prime}\right)$ exclusively, and $(\mathscr{N}, \mathscr{X})$ $\vDash \Sigma_{n}^{1}-$ Coll $+\Pi_{n}^{1}-\mathrm{CA}_{0}$. Then $\left(\mathscr{N}^{\prime}, \mathscr{X}^{\prime}\right) \vDash \Pi_{n}^{1}-\mathrm{CA}_{0}$.

Proof. Since $\Pi_{0}^{1}-\mathrm{CA}_{0}$ is just $\mathrm{ACA}_{0}$, we know that $\left(\mathscr{N}^{\prime}, \mathscr{X}^{\prime}\right) \vDash \Pi_{0}^{1}-\mathrm{CA}_{0}$. We now proceed by induction on $n$, so we assume that $n \geqslant 1$ and $\left(\mathscr{N}^{\prime}, \mathscr{X}^{\prime}\right) \vDash \Pi_{n-1}^{1}-\mathrm{CA}_{0}$, intending to show $\left(\mathscr{N}^{\prime}, \mathscr{X}^{\prime}\right) \vDash \Pi_{n}^{1}{ }^{-} \mathrm{CA}_{0}$. Suppose $\phi(x, Y)$ is $\Pi_{n}^{1}$. We want to show:

$$
\left(\mathscr{N}^{\prime}, \mathscr{X}^{\prime}\right) \vDash \forall Y \exists X \forall x\left(x \in X \leftrightarrow \phi^{\prime}(x, Y)\right) .
$$

Since the extension is exclusive, it suffices to show that for every $A \in \mathscr{X}$

$$
\left(\mathscr{N}^{\prime}, \mathscr{X}^{\prime}\right) \vDash \forall y \exists X \forall x\left(x \in X \leftrightarrow \phi^{\prime}\left(x,\left(A^{\prime}\right)_{y}\right)\right) .
$$

Using that $(\mathscr{N}, \mathscr{X}) \vDash \Pi_{n}^{1}-\mathrm{CA}_{0}$, we can find $B \in \mathscr{X}$ such that

$$
(\mathscr{N}, \mathscr{X}) \vDash \forall x y\left(\langle x, y\rangle \in B \leftrightarrow \phi\left(x,(A)_{y}\right)\right) .
$$

This sentence is $\Pi_{n+1}^{1}$, so applying Theorem 2.5,

$$
\left(\mathscr{N}^{\prime}, \mathscr{X}^{\prime}\right) \vDash \forall x y\left(\langle x, y\rangle \in B^{\prime} \leftrightarrow \phi^{\prime}\left(x,\left(A^{\prime}\right)_{y}\right)\right) .
$$

By arithmetic comprehension,

$$
\left(\mathscr{N}^{\prime}, \mathscr{X}^{\prime}\right) \vDash \forall y \exists X \forall x\left(x \in X \leftrightarrow\langle x, y\rangle \in B^{\prime}\right),
$$

so that $\left(\mathscr{N}^{\prime}, \mathscr{X}^{\prime}\right) \vDash \forall y \exists X \forall x\left(x \in X \leftrightarrow \phi^{\prime}\left(x,\left(A^{\prime}\right)_{y}\right)\right)$.

TheOREM 2.7. Suppose that $n<\omega,(\mathscr{N}, \mathscr{X}) \prec_{0}\left(\mathscr{N}^{\prime}, \mathscr{X}^{\prime}\right)$ exclusively, and $(\mathscr{N}, \mathscr{X})$ $\vDash \Sigma_{n+1}^{1}-\mathrm{AC}_{0}+\Pi_{n+1}^{1}-\mathrm{CA}_{0}$. Then $\left(\mathscr{N}^{\prime}, \mathscr{X}^{\prime}\right) \vDash \Sigma_{n+1}^{1}-\mathrm{AC}_{0}+\Pi_{n+1}^{1}-\mathrm{CA}_{0}$.

Proof. From Theorem 2.6 we know that $\left(\mathscr{N}^{\prime}, \mathscr{X}^{\prime}\right) \vDash \Pi_{n+1}^{1}-\mathrm{CA}_{0}$. Thus, we want to show that for every $\Pi_{n}^{1}$ formula $\phi(x, X, Y)$,

$$
\left(\mathscr{N}^{\prime}, \mathscr{X}^{\prime}\right) \vDash \forall Y\left[\forall x \exists X \phi^{\prime}(x, X, Y) \rightarrow \exists X \forall x \phi^{\prime}\left(x,(X)_{x}, Y\right)\right] .
$$

Because the extension is exclusive, we need only show that for every $A \in \mathscr{X}$,

$$
\left(\mathscr{N}^{\prime}, \mathscr{X}^{\prime}\right) \vDash \forall y\left[\forall x \exists X \phi^{\prime}\left(x, X,\left(A^{\prime}\right)_{y}\right) \rightarrow \exists X \forall x \phi^{\prime}\left(x,(X)_{x},\left(A^{\prime}\right)_{y}\right)\right] .
$$

Since $\left(\mathscr{N}^{\prime}, \mathscr{X}^{\prime}\right) \vDash \Pi_{n+1}^{1} \mathrm{CA}_{0}$, there are $B, C \in \mathscr{X}$ such that

$$
(\mathscr{N}, \mathscr{X}) \vDash \forall y\left[y \in B \leftrightarrow \exists X \forall x \phi\left(x,(X)_{x},(A)_{y}\right)\right],
$$

and

$$
(\mathscr{N}, \mathscr{X}) \vDash \forall x y\left[\langle x, y\rangle \in C \leftrightarrow \exists X \phi\left(x, X,(A)_{y}\right)\right]
$$

Therefore,

$$
(\mathscr{N}, \mathscr{X}) \vDash \forall y[\forall x\langle x, y\rangle \in C \rightarrow y \in B]
$$

These last three sentences are each $\Pi_{n+2}^{1}$ so by Theorem 2.5 they hold in $\left(\mathscr{N}^{\prime}, \mathscr{X}^{\prime}\right)$. Therefore, $(*)$ holds.

We next give the basic method for constructing rather classless models. For models $\mathscr{N} \prec \mathscr{M} \vDash \mathrm{PA}^{*}$, we say that $\mathscr{M}$ is a conservative extension of $\mathscr{N}$ if whenever $A \in \operatorname{Class}(\mathscr{M})$, then $A \cap N \in \operatorname{Def}(\mathscr{N})$. All conservative extensions are end extensions. The relevant form of the theorem of MacDowell and Specker [3] asserts that every model of $\mathrm{PA}^{*}$ has a proper, conservative extension. 
LEMMA 2.8 (Theorem 1.5 of [6] and Lemma 3.1 of [7]). Let $\alpha$ be an ordinal such that $\operatorname{cf}(\alpha)>\boldsymbol{\aleph}_{0}$. Suppose that $\left\langle\mathscr{N}_{\nu}: \nu \leqslant \alpha\right\rangle$ is a continuous chain of conservative extensions of models of PA*. Then $\mathscr{N}_{\alpha}$ is rather classless.

We can now obtain, for example, the following corollary.

Corollary 2.9. Suppose $T$ is a complete, consistent extension of CA + AC. Then for each $\kappa>\aleph_{0}$ there is a natural model $(\mathscr{N}, \mathscr{X})$ of $T$ of cardinality $\kappa$. Furthermore, if $\operatorname{cf}(\kappa)>\boldsymbol{\aleph}_{0}$, then $\mathscr{N}$ can be chosen to be $\kappa$-like.

Proof. Let $\alpha=\kappa$ if $\operatorname{cf}(\kappa)>\boldsymbol{\aleph}_{0}$; otherwise, let $\alpha=\kappa \cdot \omega_{1}$. Let $\left(\mathscr{N}_{0}, \mathscr{X}_{0}\right)$ be a countable model of $T$. Let $\mathscr{X}_{0}=\left\{A_{0}, A_{1}, A_{2}, \ldots\right\}$, and let $\mathscr{M}_{0}=\left(\mathscr{N}_{0}, A_{0}, A_{1}, \ldots\right)$ be a model of PA*. Using the MacDowell-Specker theorem, let $\left\langle\mathscr{M}_{\nu}: \nu \leqslant \alpha\right\rangle$ be a continuous chain of conservative extensions, with $\left|M_{\nu+1}\right|=\left|M_{\nu}\right|$. Now let $\mathscr{X}=$ $\operatorname{Def}\left(\mathscr{M}_{\alpha}\right)$, so that $\left(\mathscr{M}_{\alpha}, \mathscr{X}\right)$ is an exclusive extension of $\left(\mathscr{N}_{0}, \mathscr{X}_{0}\right)$. By Lemma 2.8 , $\mathscr{X}=\operatorname{Class}\left(\mathscr{M}_{\alpha}\right)$ so $\left(\mathscr{M}_{\alpha}, \mathscr{X}\right)$ is natural. Furthermore, by Theorem $2.5,\left(\mathscr{M}_{\alpha}, \mathscr{X}\right) \vDash T$. Clearly $\left|M_{\alpha}\right|=\kappa$, and $\mathscr{M}_{\alpha}$ is $\kappa$-like if $\operatorname{cf}(\kappa)>\boldsymbol{\aleph}_{0}$.

We end this section by giving a model-theoretic proof of a result of Sieg [10] which says that $\Sigma_{n+1}^{1}-\mathrm{AC}_{0}$ is conservative over $\Pi_{n}^{1}-\mathrm{CA}_{0}+\Sigma_{n}^{1}-\mathrm{AC}_{0}$ for $\Pi_{n+2}^{1}$ sentences. (This theorem will not be used in this paper, but we include it because of its close connection to results of this section.)

TheOrem 2.10. Suppose $(\mathscr{N}, \mathscr{X}) \vDash \Pi_{n}^{1}$-CA $\mathrm{CA}_{0}+\Sigma_{n}^{1}$-Coll, where $n<\omega$. Then there exists $\left(\mathscr{N}^{\prime}, \mathscr{X}^{\prime}\right) \vDash \Sigma_{n+1}^{1}-\mathrm{AC}_{0}$ such that $(\mathscr{N}, \mathscr{X}) \prec_{n+1}\left(\mathscr{N}^{\prime}, \mathscr{X}^{\prime}\right)$.

Proof. Let $(\mathscr{N}, \mathscr{X}) \vDash \Pi_{n}^{1}-\mathrm{CA}_{0}+\Sigma_{n}^{1}$-Coll, and let $\mathscr{X}=\left\{A_{i}: \quad i \in I\right\}$. Let $\left(\mathscr{N}^{\prime}, A_{i}^{\prime}\right)_{i \in I}$ be a sufficiently saturated elementary extension of $\left(\mathscr{N}, A_{i}\right)_{i \in I}$, and then let $\mathscr{X}^{\prime}=\operatorname{Def}\left(\left(\mathscr{N}^{\prime}, A_{i}^{\prime}\right)_{i \in I}\right)=\left\{\left(A_{i}^{\prime}\right)_{x}: x \in N^{\prime}, i \in I\right\}$ so that $\left(\mathscr{N}^{\prime}, \mathscr{X}^{\prime}\right)$ is an exclusive extension of $(\mathscr{N}, \mathscr{X})$. By Theorem 2.5, $(\mathscr{N}, \mathscr{X}) \prec_{n+1}\left(\mathscr{N}^{\prime}, \mathscr{X}^{\prime}\right)$ since $(\mathscr{N}, \mathscr{X}) \vDash \Sigma_{n}^{1}$-Coll. We now claim: $\left(\mathscr{N}^{\prime}, \mathscr{X}^{\prime}\right) \vDash \Sigma_{n+1}^{1}-\mathrm{AC}_{0}$.

To prove the claim, let $\phi(x, X, Y)$ be a $\Pi_{n}^{1}$ formula. We must show

$$
\left(\mathscr{N}^{\prime}, \mathscr{X}^{\prime}\right) \vDash \forall Y\left[\forall x \exists X \phi^{\prime}(x, X, Y) \rightarrow \exists X \forall x \phi^{\prime}\left(x,(X)_{x}, Y\right)\right] .
$$

Since the extension is exclusive, it suffices to show that for each $A \in \mathscr{X}$,

$$
\left(\mathscr{N}^{\prime}, \mathscr{X}^{\prime}\right) \vDash \forall y\left[\forall x \exists X \phi^{\prime}\left(x, X,\left(A^{\prime}\right)_{y}\right) \rightarrow \exists X \forall x \phi^{\prime}\left(x,(X)_{x},\left(A^{\prime}\right)_{y}\right)\right] .
$$

Fix some $a \in N^{\prime}$ such that $\left(\mathscr{N}^{\prime}, \mathscr{X}^{\prime}\right) \vDash \forall x \exists X \phi^{\prime}\left(x, X,\left(A^{\prime}\right)_{a}\right)$. We will show that for some $B \in \mathscr{X},\left(\mathscr{N}^{\prime}, \mathscr{X}^{\prime}\right) \vDash \forall x \exists z \phi^{\prime}\left(x,\left(B^{\prime}\right)_{z},\left(A^{\prime}\right)_{a}\right)$. So, suppose not. Then for each $B \in \mathscr{X},\left(\mathscr{N}^{\prime}, \mathscr{X}^{\prime}\right) \vDash \exists x \forall z \neg \phi^{\prime}\left(x,\left(B^{\prime}\right)_{z},\left(A^{\prime}\right)_{a}\right)$. In fact, if $B_{0}, B_{1}, \ldots, B_{k-1} \in \mathscr{X}$, then

$$
\left(\mathscr{N}^{\prime}, \mathscr{X}^{\prime}\right) \vDash \exists x \bigwedge_{i<k} \forall z \neg \phi^{\prime}\left(x,\left(B_{i}^{\prime}\right)_{z},\left(A^{\prime}\right)_{a}\right)
$$

as otherwise $B=\left\{\langle k z+i, w\rangle:\langle z, w\rangle \in B_{i}\right.$ and $\left.i<k\right\}$ would provide a contradiction. Since $(\mathscr{N}, \mathscr{X}) \vDash \Pi_{n}^{1}-\mathrm{CA}_{0}$, for each $B \in \mathscr{X}$ there is $C_{B} \in \mathscr{X}$ such that $(\mathscr{N}, \mathscr{X})$ $\vDash \forall x y z\left(\langle x, y, z\rangle \in C_{B} \leftrightarrow \neg \phi\left(x,(B)_{z},(A)_{y}\right)\right)$. This sentence is $\Pi_{n+1}^{1}$, so

$$
\left(\mathscr{N}^{\prime}, \mathscr{X}^{\prime}\right) \vDash \forall x y z\left(\langle x, y, z\rangle \in C_{B}^{\prime} \leftrightarrow \neg \phi^{\prime}\left(x,\left(B^{\prime}\right)_{z},\left(A^{\prime}\right)_{y}\right)\right) .
$$

Therefore, for each $B_{0}, B_{1}, \ldots, B_{k-1} \in \mathscr{X}$,

$$
\left(\mathscr{N}^{\prime}, \mathscr{X}^{\prime}\right) \vDash \exists x \bigwedge_{i<k} \forall z\left(\langle x, a, z\rangle \in C_{B_{i}}^{\prime}\right) \text {. }
$$


By saturation, there is $c \in N^{\prime}$ such that $\left(\mathscr{N}^{\prime}, \mathscr{X}^{\prime}\right) \vDash \forall z\left(\langle c, a, z\rangle \in C_{B}^{\prime}\right)$ for each $B \in \mathscr{X}$. Thus, $\left(\mathscr{N}^{\prime}, \mathscr{X}^{\prime}\right) \vDash \forall z \neg \phi^{\prime}\left(c,\left(B^{\prime}\right)_{z},\left(A^{\prime}\right)_{a}\right)$ for each $B \in \mathscr{X}$, so that $\left(\mathscr{N}^{\prime}, \mathscr{X}^{\prime}\right)$ $\vDash \forall X \neg \phi^{\prime}\left(c, X,\left(A^{\prime}\right)_{a}\right)$, and then $\left(\mathscr{N}^{\prime}, \mathscr{X}^{\prime}\right) \vDash \exists x \forall X \neg \phi^{\prime}\left(x, X,\left(A^{\prime}\right)_{a}\right)$. This is a contradiction.

3. Ramsey logics. The quantifier $Q^{2}$ is just one of an entire family of quantifiers of the Ramsey type, which are defined in $\$ 4$ of [5]. In this section we consider these quantifiers in the context of Peano arithmetic, giving them the "unbounded" interpretation.

For a finite sequence $s=\left\langle s_{0}, s_{1}, \ldots, s_{k}\right\rangle$ of positive integers, the Ramsey quantifier $Q^{s}$ is an $n$-place quantifier, where $n=s_{0}+s_{1}+\cdots+s_{k}$. The interpretation of the quantifier $Q^{s}$ in models of PA is defined by adjoining the following clause to the usual inductive definition of satisfaction for first-order formulas. If $\mathscr{N}$ is a model of PA, then the sentence

$$
Q^{s} x_{0,1}, x_{0,2}, \ldots, x_{0, s_{0}}, x_{1,1}, x_{1,2}, \ldots, x_{1, s_{1}}, \ldots, x_{k, s_{k}} \phi\left(x_{0,1}, \ldots, x_{k, s_{k}}\right)
$$

holds in $\mathscr{N}$ if there are unbounded subsets $X_{0}, X_{1}, \ldots, X_{k} \subseteq N$ such that whenever $a_{0,1}, a_{0,2}, \ldots, a_{0, s_{0}} \in X_{0}, \ldots, a_{k, 1}, \ldots, a_{k, s_{k}} \in X_{k}$, where $a_{i, 1}, a_{i, 2}, \ldots, a_{i, s_{i}}$ are distinct for $i \leqslant k$, then the sentence $\phi\left(a_{0,1}, \ldots, a_{k, s_{k}}\right)$ holds in $\mathscr{N}$.

We will refer to the logic formed by adjoining all the quantifiers $Q^{s}$ to first-order logic as Ramsey logic, and denote it by $\mathscr{R}$. Let $\mathscr{L}\left(Q^{s}, Q^{t}, \ldots\right)$ be the set of formulas in $\mathscr{R}$ involving no Ramsey quantifiers other than $Q^{s}, Q^{t}, \ldots$ If

$$
\phi\left(x, y_{0}, y_{1}, \ldots, y_{m-1}\right) \in \mathscr{R},
$$

then the induction axiom corresponding to $\phi(x, \bar{y})$ is the universal closure of the formula

$$
[\phi(0, \bar{y}) \wedge \forall x(\phi(x, \bar{y}) \rightarrow \phi(x+1, \bar{y}))] \rightarrow \forall x \phi(x, \bar{y}),
$$

which is also in $\mathscr{R}$. Let $\operatorname{PA}(\mathscr{R})$ consist of the usual first-order axioms for PA together with the set of induction axioms corresponding to formulas in $\mathscr{R}$. (Actually $\operatorname{PA}(\mathscr{R})$ contains some other axioms corresponding to schemes (1)-(3) in $\S 1$ of [8], but these axioms hold in any model of PA under the unbounded interpretation.) We let $\operatorname{PA}\left(Q^{s}, Q^{t}, \ldots\right)$ be $\operatorname{PA}(\mathscr{R}) \cap \mathscr{L}\left(Q^{s}, Q^{t}, \ldots\right)$. Then the system Ind, introduced by Macintyre [4], is just $\operatorname{PA}\left(Q^{2}, Q^{3}, Q^{4}, \ldots\right)$.

It was stated, somewhat misleadingly, in the Introduction of [8] that the theories Ind and $\operatorname{PA}\left(Q^{2}\right)$ were "essentially equivalent." This is true in that the models of $\operatorname{PA}\left(Q^{2}\right)$ constructed in [8] are also models of Ind. All the theorems in [8] about $\operatorname{PA}\left(Q^{2}\right)$ extend in a completely straightforward manner to theorems about Ind. However, it is unknown whether there are models of $\operatorname{PA}\left(Q^{2}\right)$ which are not models of $\operatorname{PA}\left(Q^{3}\right)$ or even of Ind. In fact, it is not even known whether there are models of $\operatorname{PA}\left(Q^{2}\right)$ which are not models of the seemingly much stronger theory $\operatorname{PA}\left(\mathscr{H}_{\mathscr{R}_{1}}\right)$. (See Definition 4.3 and Question 6.2.)

There are many instances where a quantifier $Q^{t}$ can be eliminated in favor of another quantifier $Q^{s}$. This is the case when $s=\left\langle s_{0}, s_{1}, \ldots, s_{k}\right\rangle, t=\left\langle t_{0}, t_{1}, \ldots, t_{m}\right\rangle$, and there is a function $f: m+1 \rightarrow k+1$ such that for any $i \leqslant k, \sum\left\{t_{j}: f(j)=i\right\}$ $\leqslant s_{i}$. We will show, as an example, how to eliminate $Q^{1,1}$ in favor of $Q^{2}$. Let 
$\phi(\bar{u})=Q^{1,1} x, y \psi(\bar{u}, x, y)$. Then let $\phi^{\prime}(\bar{u})$ be the formula

$$
Q^{2} x y\left[\left(x<y \rightarrow(x)_{0}<(x)_{1}<(y)_{0}\right) \wedge \psi\left(\bar{u},(x)_{0},(y)_{1}\right)\right] .
$$

Clearly, in any model $\mathscr{N}$ of PA, $\mathscr{N} \vDash \forall \bar{u}\left[\phi(\bar{u}) \leftrightarrow \phi^{\prime}(\bar{u})\right]$.

An immediate consequence of the preceding paragraph is that every model of Ind is a model of $\operatorname{PA}(\mathscr{R})$.

We recall the following theorem of Macintyre [4].

THEOREM 3.1. If $\mathscr{N} \vDash \operatorname{PA}\left(Q^{2}\right)$, then $\mathscr{N}$ is $\kappa$-like for some regular $\kappa$.

Thus, if $Q^{s}$ is not a quantifier of the form $Q^{1,1 \ldots 1}$, then every model of $\operatorname{PA}\left(Q^{s}\right)$ is $\kappa$-like for some regular $\kappa$. Notice that the quantifier $Q^{1,1}$ is the weakest of all nontrivial Ramsey quantifiers. Nevertheless, $\operatorname{PA}\left(Q^{1,1}\right)$ is still a powerful theory as Theorems 3.2 and 3.4 demonstrate.

THEOREM 3.2. If $\mathscr{N} \vDash \operatorname{PA}\left(Q^{1.1}\right)$, then $\mathscr{N}$ has strict cofinality.

Proof. Suppose there is a decreasing sequence of length $\kappa$, the first element of the sequence being $a$. Then the sentence $\psi(a)$ holds in $\mathscr{N}$, where $\psi(u)$ is the formula

$$
Q^{1,1} x, y\left[x<y \rightarrow\left((x)_{0}<(y)_{0} \wedge u \geqslant(x)_{1}>(y)_{1}\right)\right] \text {. }
$$

Conversely, if $\psi(b)$ holds in $\mathscr{N}$, then there is a decreasing sequence of length $\kappa$ whose first element is $b$. Clearly $\mathscr{N} \vDash \neg \psi(0)$ and $\mathscr{N} \vDash \forall u[\neg \psi(u) \rightarrow \neg \psi(u+1)]$. Since $\mathscr{N} \vDash \operatorname{PA}\left(Q^{1,1}\right)$, then $\mathscr{N} \vDash \forall u \neg \psi(u)$, contradicting $\mathscr{N} \vDash \psi(a)$.

Corollary 3.3. If $\mathscr{N} \vDash \operatorname{PA}\left(Q^{1,1}\right)$, then $(\mathscr{N}, \operatorname{Class}(\mathscr{N})) \vDash \operatorname{ACA}_{0}$.

PrOOF. Immediate from Proposition 2.4 and Theorem 3.2.

The notion of a weak model, used in [8], is equally applicable to $\mathscr{R}$. The following theorem extends Lemma 3.2 of [8] from models of $\operatorname{PA}\left(Q^{2}\right)$ to models of $\operatorname{PA}\left(Q^{1,1}\right)$. The proof is essentially the same, so we omit it.

Theorem 3.4. If $(\mathscr{N}, \mathscr{X}) \vDash \operatorname{PA}\left(Q^{1,1}\right)$, then $\left(\mathscr{N}, \mathscr{X}^{\prime}\right) \vDash \Pi_{1}^{1}-\mathrm{CA}_{0}$ for some $\mathscr{X}^{\prime} \subseteq \mathscr{X}$.

The set $\mathscr{X}^{\prime}$ can be the set of those classes in $\mathscr{X}$ which are definable by means of formulas in $\mathscr{L}\left(Q^{1,1}\right)$.

Our final goal in this section is to construct models of $\operatorname{PA}\left(Q^{1,1}\right)$ which are not models of $\operatorname{PA}\left(Q^{2}\right)$. The construction is very similar to the one used in [8] to obtain models of $\operatorname{PA}\left(Q^{2}\right)$, but there is a new combinatorial ingredient to replace the $\Delta$-system lemma (Lemma 3.6 below) used in [8]. For a set $A$ we will use the standard notation $[A]^{n}$ to denote the set of all $n$-element subsets of $A$. If $(A,<)$ is a linearly ordered set, $a \in[A]^{n}$, and $i<n$, then we let $a^{i}$ be the $i$ th element in increasing order of $a$.

The following lemma is very easily proved in the same manner as the $\Delta$-system lemma is, for example, in [2]. The $\Delta$-system lemma is an immediate consequence.

Lemma 3.5. Let $(A,<)$ be a linearly ordered set and $\kappa$ an infinite cardinal. Suppose that $1 \leqslant n<\omega$ and that $\left\langle a_{\nu}: \nu<\kappa\right\rangle$ is a sequence from $[A]^{n}$. Then there are $b \in A$, $m \leqslant n$, and an unbounded $I \subseteq \kappa$ such that:

(1) if $\nu \in I$ and $i<m$, then $a_{\nu}^{i} \leqslant b$;

(2) if $\mu, \nu \in I, \mu<\nu$, and $m \leqslant i<n$, then $b<a_{\mu}^{n-1}<a_{\nu}^{i}$. 
LemMA $3.6(\Delta$-system lemma $)$. Let $(A,<)$ be a $\kappa$-like linearly ordered set, where $\kappa$ is regular. Suppose that $1 \leqslant n<\omega$ and that $\left\langle a_{\nu}: \nu\langle\kappa\rangle\right.$ is a sequence from $[A]^{n}$. Then there are $m \leqslant n$ and an unbounded $I \subseteq \kappa$ such that:

(1) if $\mu, \nu \in I$ and $i<m$, then $a_{\mu}^{i}=a_{\nu}^{i}$;

(2) if $\mu, \nu \in I, \mu<\nu$, and $m \leqslant i<n$, then $a_{\mu}^{n-1}<a_{\nu}^{i}$.

Suppose $(A,<)$ is a linearly ordered set, and let $a, c \in[A]^{m}$ and $b, d \in[A]^{n}$, where $m, n<\omega$. We say that $\langle a, b\rangle$ has the same pattern as $\langle c, d\rangle$ if whenever $i<m$ and $j<n$, then $a^{i}<b^{j}$ iff $c^{i}<d^{j}$.

LemMA 3.7. Let $(A,<)$ be a linearly ordered set having strict cofinality $\kappa$. Suppose $\left\langle a_{\nu}: \nu\langle\kappa\rangle\right.$ and $\left\langle b_{\nu}: \nu\langle\kappa\rangle\right.$ are sequences from $[A]^{m}$ and $[A]^{n}$ respectively. Then there are unbounded $I, J \subseteq \kappa$ such that whenever $\mu_{0}, \mu_{1} \in I$ and $\nu_{0}, \nu_{1} \in J$, then $\left\langle a_{\mu_{0}}, b_{\nu_{0}}\right\rangle$ has the same pattern as $\left\langle a_{\mu_{1}}, b_{\nu_{1}}\right\rangle$.

Proof. We will prove the lemma by induction on $m$ and $n$. Suppose $m=n=1$. Without the loss of much generality, we can assume that both sequences $a$ and $b$ are one-to-one. Let $P$ be the intersection of all initial segments $X$ of $A$ such that $\left|\left\{\nu<\kappa: a_{\nu} \in X\right\}\right|=\kappa$. Let $Q$ be the union of all initial segments $Y$ of $A$ such that $\left|\left\{\nu<\kappa: b_{\nu} \notin Y\right\}\right|=\kappa$. We consider several cases. If there is $c \in Q-P$, then let $I=\left\{\nu<\kappa: a_{\nu}<c\right\}$ and $J=\left\{\nu<\kappa: b_{\nu}>c\right\}$. If there is $c \in P-Q$, then let $I=\left\{\nu<\kappa: a_{\nu}>c\right\}$ and $J=\left\{\nu<\kappa: b_{\nu}<c\right\}$. Now suppose $P=Q$. Then $\mid\{\nu<\kappa$ : $\left.a_{\nu} \in P\right\} \mid<\kappa$ as otherwise $\left(\left\{a_{\nu}: \nu<\kappa\right\} \cap P,<\right)$ would be $\kappa$-like. Similarly $\mid\{\nu<\kappa$ : $\left.b_{\nu} \notin P\right\} \mid<\kappa$. Thus, let $I=\left\{\nu<\kappa: a_{\nu} \notin P\right\}$ and $J=\left\{\nu<\kappa: b_{\nu} \in Q\right\}$.

Now suppose that $n>1$. For $\nu<\kappa$ let $c_{\nu}=\left\{b_{\nu}^{0}\right\}$ and $d_{\nu}=b_{\nu}-c_{\nu}$. By the inductive hypothesis, applied twice, there are unbounded $I, J \subseteq \kappa$ such that whenever $\mu_{0}, \mu_{1} \in I$ and $\nu_{0}, \nu_{1} \in J$, then $\left\langle a_{\mu_{0}}, c_{\nu_{0}}\right\rangle$ and $\left\langle a_{\mu_{0}}, d_{\nu_{0}}\right\rangle$ have the same pattern as $\left\langle a_{\mu_{1}}, c_{\nu_{1}}\right\rangle$ and $\left\langle a_{\mu_{1}}, d_{\nu_{1}}\right\rangle$ respectively. But then $\left\langle a_{\mu_{0}}, b_{\nu_{0}}\right\rangle$ has the same pattern as $\left\langle a_{\mu_{1}}, b_{\nu_{1}}\right\rangle$.

Let $\mathscr{L}$ and PA* $^{*}$ be as in $\S 2$. In $\S 2$ of [8] the $\kappa$ th iterated canonical end extension of a model $\mathscr{N}^{*}$ of PA* is constructed to obtain models of $\operatorname{PA}\left(Q^{2}\right)$. More generally, for any linearly ordered set $(I,<)$, the $I$ th iterated canonical end extension is obtained as follows. There is a set $\left\{\theta_{m}(v): m<\omega\right\}$ of $\mathscr{L}$-formulas which determines a minimal type in any complete extension of $\mathrm{PA}^{*}$. In particular, each of the following holds:

(3.A) $\mathrm{PA}^{*} \vdash \forall w \exists v>w \theta_{m}(v)$;

(3.B) $\mathrm{PA}^{*} \vdash \forall v\left(\theta_{m+1}(v) \rightarrow \theta_{m}(v)\right)$;

(3.C) for any formula $\psi\left(\bar{x}, v_{0}, v_{1}, \ldots, v_{s-1}\right)$ there is $m<\omega$ such that

$$
\begin{aligned}
\mathrm{PA}^{*} \vdash \forall \bar{x} \exists w[ & \forall \bar{v}\left(w<v_{0}<\cdots<v_{s-1} \wedge \bigwedge_{i<s} \theta_{m}\left(v_{i}\right) \rightarrow \psi(\bar{x}, \bar{v})\right) \\
& \left.\vee \forall \bar{v}\left(w<v_{0}<\cdots<v_{s-1} \wedge \bigwedge_{i<s} \theta_{m}\left(v_{i}\right) \rightarrow \neg \psi(\bar{x}, \bar{v})\right)\right] .
\end{aligned}
$$


Moreover, suppose $(I,<)$ is a linearly ordered set such that $I \cap N=\varnothing$. Then there is a conservative extension $\mathscr{M}^{*}$ of $\mathscr{N}^{*}$ where $\mathscr{M}^{*}$ is generated by $N \cup I, I$ is an ordered set of indiscernibles in $M$ over $N$, and each $a \in I$ realizes $\left\{\theta_{m}(v): m<\omega\right\}$. Furthermore, if $J$ is an initial segment of $I$, then $\mathscr{M}^{*}$ is a conservative extension of its submodel generated by $J \cup N$. We will refer to the model $\mathscr{M}^{*}$ as the $I$ th iterated canonical end extension of $\mathscr{N}^{*}$, and use it to construct models of $\operatorname{PA}\left(Q^{1,1}\right)$.

Let $(\mathscr{N}, \mathscr{X})$ be a countable model of $\Pi_{1}^{1}-\mathrm{CA}_{0}$. Then let $\mathscr{N}^{*}=$ $\left(\mathscr{N}, X_{0}, X_{1}, X_{2}, \ldots\right)$, where $\mathscr{X}=\left\{X_{0}, X_{1}, \ldots\right\}$, so that $\mathscr{N}^{*}$ is a model of $\mathrm{PA}^{*}$. Let $(I,<)$ be any linearly ordered set having strict cofinality $\kappa>\boldsymbol{\kappa}_{0}$ such that $|I|=\lambda$. Let $\mathscr{M}^{*}=\left(\mathscr{M}, Y_{0}, Y_{1}, \ldots\right)$ be the $I$ th iterated canonical end extension of $\mathscr{N}^{*}$.

Notice that Lemma 4.8 implies that $\mathscr{M}$ is rather classless, so from Theorem 2.6 $(\mathscr{M}, \operatorname{Class}(\mathscr{M})) \vDash \Pi_{1}^{1}-\mathrm{CA}_{0}$.

We now claim: $\mathscr{M} \vDash \operatorname{PA}\left(Q^{1,1}\right)$. We will prove this claim by induction on formulas, showing that if $\phi\left(x_{0}, x_{1}, \ldots, x_{n-1}, A\right)$ is a formula in $\mathscr{L}\left(Q^{1,1}\right)$ and $A \in \operatorname{Class}(\mathscr{N})$, then $\left\{\left\langle a_{0}, a_{1}, \ldots, a_{n-1}\right\rangle: \mathscr{M} \vDash \phi(\bar{a}, A)\right\}$ is a class. There is only one interesting step in the induction, which reduces to proving the following: If $A$ is a class, then $Z=\left\{a \in M: \mathscr{M} \vDash Q^{1,1} x, y(\langle x, y, a\rangle \in A)\right\}$ is a class. Let unbounded subsets $X$, $Y \subseteq M$ be called witness sets for $Q^{1,1} x, y(\langle x, y, a\rangle \in A)$ if $\mathscr{M} \vDash \forall x \in X \forall y \in Y$ $(\langle x, y, a\rangle \in A)$. We will show that if there are witness sets for $Q^{1,1} x, y(\langle x, y, a\rangle \in A)$, then there are witness sets which are classes. Therefore, since $(\mathscr{M}, \operatorname{Class}(\mathscr{M})) \vDash \Pi_{1}^{1}$ $\mathrm{CA}_{0}$, we will have that $Z \in \operatorname{Class}(\mathscr{M})$.

So, let $X$ and $Y$ be witness sets. By taking cofinal subsets of $X$ and $Y$, if necessary, there are terms $\tau_{1}\left(x_{0}, \ldots, x_{m-1}\right), \tau_{2}\left(y_{0}, \ldots, y_{n-1}\right)$ and sequences $\left\langle a_{\nu}\right.$ : $\nu\langle\lambda\rangle,\left\langle b_{\nu}: \nu\langle\lambda\rangle\right.$ of elements of $[I]^{m},[I]^{n}$ respectively such that $X=$ $\left\{\tau_{1}\left(a_{\nu}^{0}, a_{\nu}^{1}, \ldots, a_{\nu}^{m-1}\right): \nu<\kappa\right\}$ and $Y=\left\{\tau_{2}\left(b_{\nu}^{0}, b_{\nu}^{1}, \ldots, b_{\nu}^{n-1}\right): \nu<\kappa\right\}$. Applying Lemma 3.5 and then Lemma 3.7 we can assume that there are $b \in M$ and $r<m$, $s<n$ such that:

(1) if $\nu<\kappa$ and $i<m$, then $a_{\nu}^{i}<b$ iff $i<r$;

(2) if $\nu<\kappa$ and $i<n$, then $b_{\nu}^{i}<b$ iff $i<s$;

(3) if $\mu_{0}, \mu_{1}, \nu_{0}, \nu_{1}<\kappa$, then $\left\langle\left\{a_{\mu_{0}}^{0}, \ldots, a_{\mu_{0}}^{r-1}\right\},\left\{b_{\nu_{0}}^{0}, \ldots, b_{\nu_{0}}^{s-1}\right\}\right\rangle$ has the same pattern as $\left\langle\left\{a_{\mu_{1}}^{0}, \ldots, a_{\mu_{1}}^{r-1}\right\},\left\{b_{\nu_{1}}^{0}, \ldots, b_{\nu_{1}}^{s-1}\right\}\right\rangle$;

(4) $\left\langle a_{\nu}^{r}: \nu\langle\lambda\rangle\right.$ and $\left\langle b_{\nu}^{s}: \nu\langle\lambda\rangle\right.$ are cofinal in $I$.

Then there is a formula $\theta(v)$ of $\mathscr{L}^{*}$, possibly involving some parameters from $M$, such that $\mathscr{M}^{*} \vDash \forall u \exists v(u<v \wedge \theta(v))$,

$$
\begin{aligned}
\mathscr{M}^{*} \vDash \forall u_{r}, \ldots, u_{m-1}, v_{s}, \ldots, v_{n-1}\left[u_{r}\right. & <u_{r+1}<\cdots<u_{m-1}<v_{s}<v_{s+1} \\
& <\cdots<v_{n-1} \wedge \theta\left(u_{r}\right) \wedge \ldots \\
& \wedge \theta\left(u_{m-1}\right) \wedge \theta\left(v_{s}\right) \wedge \cdots \wedge \theta\left(v_{n-1}\right) \\
\rightarrow & \left\langle\tau_{1}\left(a_{0}^{0}, a_{0}^{1}, \ldots, a_{0}^{r-1}, u_{r}, u_{r+1}, \ldots, u_{m-1}\right),\right. \\
& \left.\left.\tau_{2}\left(b_{0}^{0}, b_{0}^{1}, \ldots, b_{0}^{s-1}, v_{s}, v_{s+1}, \ldots, v_{n-1}\right), a\right\rangle \in A\right],
\end{aligned}
$$


and

$$
\begin{aligned}
\mathscr{M}^{*} \vDash \forall u_{r}, \ldots, u_{m-1}, v_{s}, \ldots, v_{n-1}\left[v_{s}<\right. & v_{s+1}<\cdots<v_{n-1}<u_{r}<u_{r+1} \\
& <\cdots<u_{m-1} \wedge \theta\left(u_{r}\right) \wedge \cdots \wedge \theta\left(u_{m-1}\right) \\
& \wedge \theta\left(v_{s}\right) \wedge \cdots \wedge \theta\left(v_{n-1}\right) \\
\rightarrow & \left\langle\tau_{1}\left(a_{0}^{0}, \ldots, a_{0}^{r-1}, u_{r}, \ldots, u_{m-1}\right),\right. \\
& \left.\left.\tau_{2}\left(b_{0}^{0}, \ldots, b_{0}^{s-1}, v_{s}, \ldots, v_{n-1}\right), a\right\rangle \in A\right] .
\end{aligned}
$$

Let $g(z)$ be a term denoting the $z$ th element satisfying $\theta(v)$. Let $k=m+n-(r+$ $s)$ and set

$$
X_{0}=\left\{\tau_{1}\left(a_{0}^{0}, a_{0}^{1}, \ldots, a_{0}^{r-1}, g(k z), g(k z+1), \ldots, g(k z+m-r-1)\right): z \in M\right\}
$$

and

$$
\begin{aligned}
Y_{0}=\left\{\tau _ { 2 } \left(b_{0}^{0}, b_{0}^{1}, \ldots, b_{0}^{s-1}, g(k z+m-r)\right.\right. & , \ldots, \\
& \quad g(k z+m+n-r-s-1)): z \in M\} .
\end{aligned}
$$

Clearly, $X_{0}, Y_{0} \in \operatorname{Class}(\mathscr{M})$, and $X_{0}, Y_{0}$ are witness sets for $Q^{1,1} x, y(\langle x, y, a\rangle \in A)$.

This completes the demonstration that $\mathscr{M}$ is a model of $\operatorname{PA}\left(Q^{1,1}\right)$. We remark that it can be shown that $\mathscr{M}$ is a model of $\operatorname{PA}\left(Q^{1,1, \ldots, 1}\right)$. In fact, we know of no model of $\operatorname{PA}\left(Q^{1,1}\right)$ which is not a model of $\operatorname{PA}\left(Q^{1,1,1}\right)$ or even of $\operatorname{PA}\left(Q^{1,1}, Q^{1,1,1}, \ldots\right)$. We also remark that if the ordered set $(I,<)$ occurring in the construction of $\mathscr{M}$ is $\kappa$-like for some uncountable, regular $\kappa$, then $\mathscr{M}$ is a model of $\operatorname{PA}\left(Q^{2}\right)$. (See Questions 6.3 and 6.4.)

The above construction of models of $\operatorname{PA}\left(Q^{1,1}\right)$, together with Theorem 3.2, yields several corollaries.

COROLLARY 3.8. For any infinite cardinal $\kappa$, the following are equivalent:

(1) there is a model of $\operatorname{PA}\left(Q^{1.1}\right)$ of cardinality $\kappa$;

(2) $\kappa$ is not a singular, strong limit cardinal;

(3) there is a linearly ordered set $(I,<)$ of cardinality $\kappa$ having strict cofinality.

Proof. (1) $\Rightarrow$ (3) by Theorem 3.2 .

(3) $\Rightarrow(1)$. If $\kappa=\aleph_{0}$, let $\mathscr{M}$ be the standard model. If $\kappa>\boldsymbol{\aleph}_{0}$, use the above construction of $\mathscr{M}$.

$(3) \Rightarrow(2)$. Let $(I,<)$ have strict cofinality $\lambda$. If $\kappa$ is a singular, strong limit cardinal, then $\left(2^{\lambda}\right)^{+}<\kappa$. Therefore, for some $a \in I, B=\{x \in I: x<a\}$ has cardinality $\geqslant\left(2^{\lambda}\right)^{+}$. From the Erdös-Rado Theorem, there is a subset $X \subseteq B$ of cardinality $\lambda^{+}$which is either well-ordered or inversely well-ordered. This contradicts $(I,<)$ having strict cofinality.

$(2) \Rightarrow(3)$. If $\kappa$ is regular, then let $(I,<)$ be any $\kappa$-like linear order. If $\kappa$ is singular but not a strong limit cardinal, then there is $\lambda<\kappa$ such that $2^{\lambda} \geqslant \kappa$. It is well known that there is a linearly ordered set $(A,<)$ such that $|A|=\kappa$ and $A$ has no subset of cardinality $\lambda^{+}$which is well-ordered or inversely well-ordered. Suppose $A$ has order type $\alpha$. Then let $(I,<)$ be a linearly ordered set which has ordered type $\alpha \cdot \lambda^{+}$. 
The next two corollaries are proved similarly. The first one of them shows that there is a model of $\operatorname{PA}\left(Q^{1,1}\right)$ of cardinality $\aleph_{1}$ which is not a model of $\operatorname{PA}\left(Q^{2}\right)$.

COROLlaRY 3.9. For any uncountable cardinal $\kappa$, the following are equivalent:

(1) there is a model of $\operatorname{PA}\left(Q^{1,1}\right)$ of cardinality $\kappa$ which is not $\kappa$-like (and, therefore, not a model of $\left.\mathrm{PA}\left(Q^{2}\right)\right)$;

(2) $\kappa$ is not a singular, strong limit cardinal and is not weakly compact;

(3) there is a linearly ordered set of cardinality $\kappa$ which is not $\kappa$-like but which has strict cofinality.

COROLlaRY 3.10. For any infinite cardinal $\kappa$, the following are equivalent:

(1) there is a $\kappa$-like model of $\operatorname{PA}\left(Q^{1,1}\right)$;

(2) $\kappa<2^{\mathrm{cf}(\kappa)}$

(3) there is a $\kappa$-like linearly ordered set having strict cofinality.

For a related open question, see Question 6.4.

4. Hyper-Ramsey logic. The intended interpretation of the quantifier $Q^{n}$ is rendered by the following clause, which is added to the usual inductive definition of satisfaction for first-order formulas to yield the definition of satisfaction for Ramsey formulas. If $\mathscr{N}$ is a model of PA, then the sentence $Q^{n} x_{1} x_{2} \cdots x_{n} \phi\left(x_{1}, x_{2}, \ldots, x_{n}\right)$ holds in $\mathscr{N}$ iff there is an unbounded subset $X \subseteq N$ such that for any distinct $a_{1}$, $a_{2}, \ldots, a_{n} \in X$, the sentence $\phi\left(a_{1}, a_{2}, \ldots, a_{n}\right)$ holds in $\mathscr{N}$. It is often more natural and convenient (as, for example, in $\$ 4$ of [8]) to give $Q^{n}$ the order interpretation in which $Q^{n} x_{1} x_{2} \cdots x_{n} \phi\left(x_{1}, x_{2}, \ldots, x_{n}\right)$ holds iff there is an unbounded $X$ such that for any increasing $n$-tuple $a_{1}<a_{2}<\cdots<a_{n}$ of elements from $X, \phi\left(a_{1}, a_{2}, \ldots, a_{n}\right)$ holds in $\mathscr{N}$. There is no significant difference between these two interpretations of $Q^{\prime \prime}$. The latter interpretation can be described in another manner which has the advantage of suggesting the generalization of Ramsey logic which will subsequently be presented. This description is best given in terms of second-order logic.

Some routine abbreviations will be useful. First, let $Q x \cdots$ be an abbreviation for $\forall w \exists x(x>w \wedge \cdots)$. All the other abbreviations are given in terms of $Q$ and are indicated below, where the formula in the second column is abbreviated by the corresponding entry in the first column.

$$
\begin{array}{ll}
Q^{*} x \cdots & \neg Q x \neg \cdots, \\
Q x \in X \cdots & Q x(x \in X \wedge \cdots), \\
Q^{*} x \in X \cdots & Q^{*} x(x \in X \rightarrow \cdots), \\
\exists^{\infty} X \cdots & \exists X((Q x x \in X) \wedge \cdots), \\
\forall^{\infty} X \cdots & \forall X((Q x x \in X) \rightarrow \cdots) .
\end{array}
$$

The formula $Q^{n} x_{1} x_{2} \cdots x_{n} \phi\left(x_{1}, x_{2}, \ldots, x_{n}\right)$, in the order interpretation, is now easily seen to be equivalent to the formula

$$
\exists^{\infty} X Q^{*} x_{1} \in X \cdots Q^{*} x_{n} \in X \phi\left(x_{1}, x_{2}, \ldots, x_{n}\right),
$$

while its negation is equivalent to

$$
\forall^{\infty} X Q x_{1} \in X \cdots Q x_{n} \in X \neg \phi\left(x_{1}, x_{2}, \ldots, x_{n}\right) .
$$


Motivated in part by the syntactic form of these two second-order formulas, we define a syntactic fragment of second-order logic, which we call hyper-Ramsey logic and denote by $\mathscr{H} \mathscr{R}_{\omega}$. Although hyper-Ramsey logic is a fragment of second-order logic, the intention is for it to be regarded as an extended first-order logic in the same way that Ramsey logic is a first-order logic.

Definition 4.1. The set $\mathscr{H} \mathscr{R}$ is the smallest set of second-order formulas satisfying the following requirements:

(0) All first-order formulas are in $\mathscr{H} \mathscr{R}$.

(1) If $\phi_{1}$ and $\phi_{2}$ are in $\mathscr{H} \mathscr{R}$, then so are $\phi_{1} \wedge \phi_{2}, \phi_{1} \vee \phi_{2}, \neg \phi_{1}, \exists x \phi_{1}$, and $\forall x \phi_{1}$.

(2) If $\phi$ is in $\mathscr{H} \mathscr{R}$ and has no free second-order variables other than possibly $X$, and all free occurrences of $X$ in $\phi$ are positive, then $Q x \in X \phi$ is in $\mathscr{H} \mathscr{R}$.

$\left(2^{*}\right)$ If $\phi$ is in $\mathscr{H} \mathscr{R}$ and has no free second-order variables other than possibly $X$, and all free occurrences of $X$ in $\phi$ are negative, then $Q^{*} x \in X \phi$ is in $\mathscr{H} \mathscr{R}$.

(3) If $\phi$ is in $\mathscr{H} \mathscr{R}$ and all free occurrences of $X$ in $\phi$ are positive (and occur in the form $Q x \in X)$, then $\forall^{\infty} X \phi$ is in $\mathscr{H} \mathscr{R}$.

(3*) If $\phi$ is in $\mathscr{H} \mathscr{R}$ and all free occurrences of $X$ in $\phi$ are negative (and occur in the form $Q^{*} x \in X$ ), then $\exists^{\infty} X \phi$ is in $\mathscr{H} \mathscr{R}$.

Notice that $\left(2^{*}\right)$ and $\left(3^{*}\right)$ are superfluous in that $Q^{*} x \in X \phi$ is equivalent to $\neg Q x \in X \neg \phi$ and $\exists^{\infty} X \phi$ is equivalent to $\neg \forall^{\infty} X \neg \phi$. Dually, (2) and (3) may instead by viewed as superfluous. The parenthetical phrases in (3) and (3*) are redundant.

Since it is intended that this logic be an extended first-order logic, we want to single out those formulas of $\mathscr{H} \mathscr{R}$ which have no free second-order variables.

Definition 4.2. Let $\mathscr{H} \mathscr{R}_{\omega}$ be the set of formulas in $\mathscr{H} \mathscr{R}$ which have no free second-order variables. For each $n<\omega$ let $\mathscr{H} \mathscr{R}_{n}$ be the set of those formulas $\phi$ in $\mathscr{H} \mathscr{R}_{\omega}$ such that $\lambda(\phi) \leqslant n$. (See Definition 1.2.)

Notice that $\mathscr{H}_{0}$ is just the set of first-order formulas.

If $\phi\left(x, y_{0}, y_{1}, \ldots, y_{m-1}\right) \in \mathscr{H} \mathscr{R}_{\omega}$, then the induction axiom corresponding to $\phi(x, \bar{y})$ is the universal closure of the formula

$$
\phi(0, \bar{y}) \wedge \forall x[\phi(x, \bar{y}) \rightarrow \phi(x+1, \bar{y})] \rightarrow \forall x \phi(x, \bar{y}),
$$

which also is in $\mathscr{H} \mathscr{R}_{\omega}$. More specifically if $\phi \in \mathscr{H} \mathscr{R}_{n}$, then its corresponding induction axiom is also in $\mathscr{H} \mathscr{R}_{n}$.

Definition 4.3. The system $\operatorname{PA}\left(\mathscr{H}_{\mathscr{R}_{n}}\right)$, for $n \leqslant \omega$, consists of the usual first-order axioms for PA together with the set of induction axioms corresponding to formulas in $\mathscr{H}_{n}$.

Obviously, if $m \leqslant n \leqslant \omega$, then $\operatorname{PA}=\operatorname{PA}\left(\mathscr{H} \mathscr{R}_{0}\right) \subseteq \operatorname{PA}\left(\mathscr{H} \mathscr{R}_{m}\right) \subseteq \operatorname{PA}\left(\mathscr{H} \mathscr{R}_{n}\right)$ and $\operatorname{PA}\left(\mathscr{H} \mathscr{R}_{\omega}\right)=\bigcup\left\{\operatorname{PA}\left(\mathscr{H} \mathscr{R}_{k}\right): k<\omega\right\}$. The logic $\mathscr{H} \mathscr{R}_{1}$ is seen to extend logic with the Ramsey quantifiers. The following lemma is easily proved by an induction on formulas.

LEMMA 4.4. Let $\phi(\bar{u})$ be a formula in the language of $\operatorname{PA}\left(Q^{2}, Q^{3}, Q^{4}, \ldots\right)$ (with the order interpretation), and let $\phi^{\prime}$ be the formula in $\mathscr{H}_{1}$ derived from $\phi$ by replacing each occurrence of $Q^{n} x_{1} x_{2} x_{3} \cdots$ by $\exists^{\infty} X Q^{*} x_{1} \in X Q^{*} x_{2} \in X \cdots$. Then $\phi^{\prime} \in \mathscr{H} \mathscr{R}_{1}$, and if $\mathcal{N} \vDash \mathrm{PA}$, then $\mathcal{N} \vDash \forall \bar{u}\left(\phi(\bar{u}) \leftrightarrow \phi^{\prime}(\bar{u})\right)$. 
Corollary 4.5. If $\mathscr{N}$ is a model of $\operatorname{PA}\left(\mathscr{H} \mathscr{R}_{1}\right)$, then $\mathscr{N}$ is a model of $\operatorname{PA}\left(Q^{2}\right)$ and, consequently, is $\kappa$-like for some regular $\kappa$.

Proof. This is immediate from Lemma 4.4 and Theorem 3.1.

LEMMA 4.6. If $n \leqslant \omega$ and $(\mathscr{N}, \mathscr{X}) \vDash \Lambda_{n}^{1}-\mathrm{CA}_{0}$, then $(\mathscr{N}, \mathscr{X}) \vDash \operatorname{PA}\left(\mathscr{H} \mathscr{R}_{n}\right)$.

Proof. Let $\phi(x, \bar{y})$ be a formula in $\operatorname{PA}\left(\mathscr{H}_{\mathscr{R}_{n}}\right)$, and let $\bar{b} \in N$ be such that $(\mathscr{N}, \mathscr{X}) \vDash \phi(0, \bar{b}) \wedge \forall x(\phi(x, \bar{b}) \rightarrow \phi(x+1, \bar{b}))$. Since $\phi(x, \bar{y}) \in \Lambda_{n}^{1}$, there is $A \in$ $\mathscr{X}$ such that $\forall x(x \in A \leftrightarrow \phi(x, \bar{b}))$. It follows from $A$ being a class that $A=N$. Hence, $(\mathscr{N}, \mathscr{X}) \vDash \forall x \phi(x, \bar{b})$, thereby proving the induction axiom corresponding to $\phi(x, \bar{y})$.

Corollary 4.7. If $n<\omega$ and $(\mathscr{N}, \mathscr{X}) \vDash \Pi_{n+1}^{1}-\mathrm{CA}_{0}+\sum_{n}^{1}-\mathrm{AC}_{0}$, then $(\mathscr{N}, \mathscr{X}) \vDash$ $\operatorname{PA}\left(\mathscr{H}_{n+1}\right)$.

Proof. This follows immediately from Lemma 4.6 and Lemma 1.3.

The logic $\mathscr{H} \mathscr{R}_{\omega}$ is very expressive. To see an example of this we will consider what appears to be an even more expressive logic, and then show that it possesses the same expressibility as $\mathscr{H} \mathscr{R}_{\omega}$. We add to the language a new second-order unary predicate symbol $C$. The intended interpretation of the formula $C(X)$ in the model $\mathscr{N}$ is that $X \in \operatorname{Class}(\mathscr{N})$. Now we consider the set $\mathscr{H} \mathscr{R}^{C}$ of formulas which has the same defining properties as $\mathscr{H} \mathscr{R}$ in Definition 4.1 but with the following additional properties:

(4) $x \in X$ is in $\mathscr{H}^{C}$ for each first- and second-order variables $x$ and $X$ respectively.

(5) If $\phi \in \mathscr{H} \mathscr{R}^{C}$, then both $\exists X(C(X) \wedge \phi)$ and $\forall X(C(X) \rightarrow \phi)$ are in $\mathscr{H}^{C}$.

Notice that the parenthetical phrases in (3) and $\left(3^{*}\right)$ are now essential.

For $n \leqslant \omega$, let $\mathscr{H}_{n} \mathscr{R}_{n}^{C}$ be the set of those formulas $\phi$ in $\mathscr{H}^{C}$ without free second-order variables such that $\lambda(\phi) \leqslant n$.

Proposition 4.8. With each formula $\phi(\bar{u})$ in $\mathscr{H}_{R_{n}}^{C}$, there is effectively associated a formula $\hat{\phi} \in \mathscr{H} \mathscr{R}_{n}$ having the same free variables as $\phi$ such that for any model $\mathscr{N}$ of PA, $\mathscr{N} \vDash \forall \bar{u}(\phi(\bar{u}) \leftrightarrow \hat{\phi}(\bar{u}))$.

Proof. Define $\hat{\phi}$ inductively on subformulas; the only step not commuting with formula formation occurs when $\phi$ has the form $\exists X(C(X) \wedge \theta)$ or $\forall X(C(X) \rightarrow \theta)$. By duality, we need only consider one of these, so let us consider the first one. Let $x \in y$ be an abbreviation for the formula asserting "the $x$ th element of the finite sequence of 0 's and 1's coded by $y$ is a 1," and let $y \triangleleft z$ be an abbreviation for the formula asserting “the sequence of 0 's and 1's coded by $y$ is a proper initial segment of the one coded by $z$." Let $\theta^{\prime}$ be derived from $\hat{\theta}$ by replacing all negative occurrences of formulas of the form $v \in X$ by $Q x \in X v \in X$ and all positive occurrences by $Q^{*} x \in X v \in x$. Then let $\hat{\phi}$ be $\exists^{\infty} X Q^{*} x \in X Q^{*} y \in X\left(x \triangleleft y \wedge \theta^{\prime}\right)$. It is easily verified that $\hat{\phi} \in \mathscr{H} \mathscr{R}_{n}$ and $\mathscr{N} \vDash \forall \bar{u}(\phi(\bar{u}) \leftrightarrow \hat{\phi}(\bar{u}))$.

Corollary 4.9. Suppose $1 \leqslant n<\omega$ and $\mathscr{N} \vDash \operatorname{PA}\left(\mathscr{H}_{n}\right)$. Then $(\mathscr{N}, \operatorname{Class}(\mathscr{N}))$ $\vDash \Lambda_{n}^{1}-\mathrm{CA}_{0}$. 
Proof. It follows from Corollary 4.5 and Proposition 2.4 that $(\mathscr{N}, \operatorname{Class}(\mathscr{N})) \vDash$ $\mathrm{ACA}_{0}$.

Now let $\psi(x, \bar{y}) \in \Lambda_{n}^{1}$, and let $\phi(x, \bar{y})$ be obtained from $\psi$ by relativizing all second-order variables to $C$. By Proposition 4.8 for any $a, \bar{b} \in N,(\mathscr{N}, \operatorname{Class}(\mathscr{N}))$ $\vDash \psi(a, \bar{b})$ iff $\mathscr{N} \vDash \phi(a, \bar{b})$ iff $\mathscr{N} \vDash \phi^{\prime}(a, \bar{b})$, where $\phi^{\prime}(x, \bar{y}) \in \mathscr{H} \mathscr{R}_{n}$ is the formula from Proposition 4.8. Since $\mathscr{N} \vDash \operatorname{PA}\left(\mathscr{H}_{\mathscr{R}_{n}}\right)$, there is an $A \in \operatorname{Class}(\mathscr{N})$ such that $A=\left\{x \in N: \mathscr{N} \vDash \phi^{\prime}(x, \bar{b})\right\}$. But then also $A=\{x \in N:(\mathscr{N}, \operatorname{Class}(\mathscr{N})) \vDash$ $\psi(x, \bar{b})\}$, so that $(\mathscr{N}, \operatorname{Class}(\mathscr{N})) \vDash \exists X \forall x(x \in X \leftrightarrow \psi(x, \bar{b}))$.

Corollary 4.10. Suppose $1 \leqslant n<\omega$ and $\mathscr{N} \vDash \operatorname{PA}\left(\mathscr{H} \mathscr{R}_{n}\right)$. Then there is $X \subseteq$ $\operatorname{Class}(\mathscr{N})$ such that $(\mathscr{N}, \mathscr{X}) \vDash \Pi_{n}^{1}-\mathrm{CA}_{0}$.

Proof. This follows from Corollary 4.9 and Theorem 1.5.

5. Constructing the models. This section contains a proof of the existence of models of PA $\left(\mathscr{H} \mathscr{R}_{n}\right)$. We will be able to conclude from Theorem 5.1 and Corollary 4.10 that the first-order consequences of $\operatorname{PA}\left(\mathscr{H} \mathscr{R}_{n}\right)$ are precisely the same as the first-order consequences of $\Pi_{n}^{1}-\mathrm{CA}_{0}$.

Theorem 5.1. Suppose $n \leqslant \omega$ and $(\mathscr{M}, \mathscr{X}) \vDash \Pi_{n}^{1}-\mathrm{CA}_{0}$, and suppose $\kappa$ is a regular uncountable cardinal. Then there is a $\kappa$-like $\mathscr{N} \equiv \mathscr{M}$ such that $\mathscr{N} \vDash \operatorname{PA}\left(\mathscr{H} \mathscr{R}_{n}\right)$.

Proof. Clearly, we can suppose $n \geqslant 1$. We will also assume that $n<\omega$. To justify this last assumption we note that the construction of the model $\mathscr{N}$ will be done uniformly in $n$. Thus, if the original model $(\mathscr{M}, \mathscr{X})$ is a model $\Pi_{n}^{1}-\mathrm{CA}_{0}$ for each $n<\omega$, then the constructed model $\mathscr{N}$ will be a model of $\operatorname{PA}\left(\mathscr{H} \mathscr{R}_{n}\right)$ for each $n<\omega$.

Without loss of generality we can suppose that both $\mathscr{M}$ and $\mathscr{X}$ are countable, and by Theorem 1.4 we can assume that $(\mathscr{M}, \mathscr{X}) \vDash \Pi_{n}^{1}-\mathrm{CA}_{0}+\Sigma_{n}^{1}-\mathrm{AC}_{0}$. Let $\mathscr{X}=$ $\left\{A_{0}, A_{1}, A_{2}, \ldots\right\}$, and consider the first-order structure $\mathscr{M}^{*}=\left(\mathscr{M}, A_{0}, A_{1}, A_{2}, \ldots\right)$, which is a model of $\mathrm{PA}^{*}$. We will use $\mathscr{H} \mathscr{R}^{*}$ and $\mathscr{H}_{n}^{*}$ to apply to the language of $\mathscr{M}^{*}$.

Let $(I,<)$ be a $\kappa$-like linearly ordered set. As in $\S 3$, let $\mathscr{N}^{*}$ be the $I$ th iterated canonical end extension of $\mathscr{M}^{*}$. Recall that associated with this model is a sequence $\left\langle\theta_{m}(v): m<\omega\right\rangle$ of formulas in the language of $\mathscr{N}^{*}$ satisfying (3.A), (3.B), and (3.C) in §3. Then, it follows from Theorem 2.7 and Lemma 2.8 that

(1) $(\mathscr{N}, \operatorname{Class}(\mathscr{N})) \vDash \Pi_{n}^{1}-\mathrm{CA}_{0}+\Sigma_{n}^{1}-\mathrm{AC}_{0}$.

From Lemmas 1.3 and 4.6 we get

(2) $(\mathscr{N}, \operatorname{Class}(\mathscr{N})) \vDash \operatorname{PA}\left(\mathscr{H} \mathscr{R}_{n}\right)$.

Property (3.C) has an important consequence for which some definitions are useful. It will be necessary to be precise about exactly what the first-order variables of our logic are. So, to the existing first-order variables $x_{0}, x_{1}, x_{2}, \ldots$ let us add a supply of fresh first-order variables $u, v_{0}, v_{1}, v_{2}, \ldots$ A first-order term $\tau\left(u, v_{0}, v_{1}, \ldots, v_{s-1}\right)$ in the language of $\mathscr{N}^{*}$, but with only the displayed variables occurring freely, will be called a $(1+s)$-term. Now suppose that $A \subseteq N, \tau(u, \bar{v})$ is a $(1+s)$-term, $m<\omega$, and $b \in N$. Then we say that $A$ satisfies $\langle\tau(b, \bar{v}), m\rangle$ if there is a set $G \subseteq[N]^{s}$ of cardinality $\kappa$ such that whenever $g, h \in G$ are distinct, then 
either $h^{s-1}<g^{0}$ or $g^{s-1}<h^{0}$, and $A=\left\{\tau\left(b, g^{0}, g^{1}, \ldots, g^{s-1}\right): g \in G\right\}$, and such that $\mathscr{N}^{*} \vDash \theta_{m}\left(g^{i}\right)$ whenever $g \in G$ and $i<s$. From Lemma 3.6 we obtain the following:

(3) If $A \subseteq N$ is unbounded, then there is an unbounded $D \subseteq A$, a $(1+r)$-term $\tau(u, \bar{v})$ for some $r<\omega$, and an element $b \in N$ such that $D$ satisfies $\langle\tau(b, \bar{v}), m\rangle$ for each $m<\omega$.

We finally note the following obvious fact:

(4) If $A \subseteq N$ is unbounded and satisfies $\langle\tau(b, \bar{v}), m\rangle$, then there is an unbounded $B \in \operatorname{Class}(\mathcal{N})$ which satisfies $\langle\tau(b, \bar{v}), m\rangle$.

Our object now is to prove the following lemma.

LEMMA 5.2. Let $\phi\left(x_{0}, x_{1}, \ldots, x_{k-1}\right)$ be any formula in $\mathscr{H}^{*}$ and let $a_{0}, a_{1}, \ldots, a_{k-1} \in N$. Then $\mathscr{N}^{*} \vDash \phi(\bar{a})$ iff $\left(\mathscr{N}^{*}, \operatorname{Class}(\mathscr{N})\right) \vDash \phi(\bar{a})$.

To see why this lemma will suffice to prove Theorem 5.1, consider some formula $\phi(x, \bar{y})$ in $\mathscr{H} \mathscr{R}_{n}$ with the intent of showing that the induction axiom corresponding to $\phi(x, \bar{y})$ holds in $\mathscr{N}$. Let $\bar{b} \in N$. Then from (2) we see that $\left(\mathscr{N}^{*}, \operatorname{Class}(\mathscr{N})\right) \vDash$ $\exists X \forall x(x \in X \leftrightarrow \phi(x, \bar{b}))$. Let $A \in \operatorname{Class}(\mathscr{N})$ witness this sentence. By the lemma, for each $a \in N, \mathscr{N}^{*} \vDash \phi(a, \bar{b})$ iff $a \in A$. But then, since $A$ is a class, if $\mathscr{N}^{*} \vDash$ $\phi(0, \bar{b}) \wedge \forall x(\phi(x, \bar{b}) \rightarrow \phi(x+1, \bar{b}))$, we get that $A=N$.

Now, to prove the lemma, we will need a definition. Suppose we are given a $(1+s)$-term $\tau(u, \bar{v})$ and a second-order variable $X$. To each formula $\phi$ in $\mathscr{H}^{*}$ which is a subformula of a formula in $\mathscr{H}_{R_{n}}^{*}$, with $X$ not occurring as a bound variable in $\phi$, we will associate a formula $\phi_{X, \tau}$. The formula $\phi_{X, \tau}$ will have the following properties:

(5) The free first-order variables of $\phi_{X, \tau}$ will be just those of $\phi$ together with $u$.

(6) The free second-order variables of $\phi_{X, \tau}$ will be just those of $\phi$ except for $X$.

(7) All quantified second-order variables of $\phi_{X, \tau}$ are relativized to $C$ (see $\S 4$ ).

(8) $\lambda\left(\phi_{X . \tau}\right) \leqslant \lambda(\phi)$ (see Definition 1.2).

Simultaneously with defining $\phi_{X, \tau}$ we will also define the natural number $m(\phi, X, \tau)$.

There is really only one interesting case in the definition. We first dispose of the uninteresting ones. If $\phi$ is atomic, then $\phi_{X, \tau}=\phi$ and $m(\phi, X, \tau)=0 ;(\neg \phi)_{X, \tau}=$ $\neg\left(\phi_{X, \tau}\right)$ and $m(\neg \phi, X, \tau)=m(\phi, X, \tau) ;(\phi \vee \psi)_{X, \tau}=\phi_{X, \tau} \vee \psi_{X, \tau}$ and

$$
m(\phi \vee \psi, X, \tau)=\max (\{m(\phi, X, \tau), m(\psi, X, \tau)\}) ;
$$

$(\exists x \phi)_{X, \tau}=\exists x\left(\phi_{X, \tau}\right)$ and $m(\exists x \phi, X, \tau)=m(\phi, X, \tau) ; \quad\left(Q^{*} x \in Y \phi\right)_{X, \tau}=Q^{*} x \in$ $Y\left(\phi_{X, \tau}\right)$ and $m\left(Q^{*} x \in Y \phi, X, \tau\right)=m(\phi, X, \tau)$, where $X$ and $Y$ are distinct variables; $\left(\exists^{\infty} Y \phi\right)_{X, \tau}=\exists^{\infty} Y\left(C(Y) \wedge \phi_{X, \tau}\right)$ and $m\left(\exists^{\infty} Y \phi, X, \tau\right)=m(\phi, X, \tau)$. Notice in this last case that $X$ and $Y$ must be distinct variables.

The interesting case in the definition of $\phi_{X, \tau}$ occurs for formulas of the form $Q^{*} x \in X \phi$. Since this formula is in $\mathscr{H} \mathscr{R}, X$ is the only free second-order variable in $\phi$. So by (6) above, there are no free second-order variables in $\phi_{X, \tau}$. From (7), (8), and (2), there is a first-order formula $\psi(x, \bar{y}, u)$ in the language of $\mathscr{N}^{*}$ such that 
$\mathscr{N}^{*} \vDash \forall x \forall \bar{y} \forall u\left(\psi \leftrightarrow \phi_{X, \tau}\right)$. By (1) there is $m<\omega$ such that

$$
\begin{array}{r}
\mathscr{N}^{*} \vDash \forall u \forall \bar{y}\left[\exists w \forall \bar{v}\left(w<v_{0}<v_{1}<\cdots<v_{s-1} \wedge \bigwedge_{i<s} \theta_{m}\left(v_{i}\right) \rightarrow \psi(\tau(u, \bar{v}), \bar{y}, u)\right)\right. \\
\left.\vee \exists w \forall \bar{v}\left(w<v_{0}<v_{1}<\cdots<v_{s-1} \wedge \bigwedge_{i<s} \theta_{m}\left(v_{i}\right) \rightarrow \neg \psi(\tau(u, \bar{v}), \bar{y}, u)\right)\right] .
\end{array}
$$

Let $m\left(Q^{*} x \in X \phi, X, \tau\right)$ be the least such $m$, and then set

$$
\begin{aligned}
\left(Q^{*} x\right. & \in X \phi)_{X, \tau} \\
& =\exists w \forall \bar{v}\left(w<v_{0}<v_{1}<\cdots<v_{s-1} \wedge \bigwedge_{i<s} \theta_{m}\left(v_{i}\right) \rightarrow \psi(\tau(u, \bar{v}), \bar{y}, u)\right) .
\end{aligned}
$$

It is obvious that if $X$ does not actually occur in $\phi$, then for any $\tau, \phi_{X, \tau}$ is just the relativization of $\phi$ to $C$. Thus, we complete the proof of the lemma by proving the following claim, which was both formulated and proved by Kaufmann.

Claim. Let $X$ be a second-order variable, and

$$
\phi\left(X_{0}, X_{1}, \ldots, X_{k-1}, x_{0}, x_{1}, \ldots, x_{l-1}, X\right)
$$

a formula in $\mathscr{H} \mathscr{R}$ which is a subformula of a formula in $\mathscr{H} \mathscr{R}_{n}$, with $X$ not an occurring bound in $\phi$. Let $\tau(u, \bar{v})$ be a $(1+s)$-term, so that $\phi_{X, \tau}=\phi_{X, \tau}(\bar{X}, \bar{x}, u)$. Let $a_{0}, a_{1}, \ldots, a_{l-1}, b \in N$, and $A_{0}, A_{1}, \ldots, A_{k-1}, B \subseteq N$, such that $B$ satisfies $\langle\tau(b, \bar{v}), m(\phi, X, \tau)\rangle$. Then $\mathscr{N}^{*} \vDash \phi(\bar{A}, \bar{a}, B)$ iff $\mathscr{N}^{*} \vDash \phi_{X, \tau}(\bar{A}, \bar{a}, b)$.

The proof of the claim proceeds by induction on $\phi$. All cases are trivial except perhaps for the cases in which the formula has form either $\exists^{\infty} Y \phi$ or $Q^{*} x \in X \phi$.

Suppose $\mathscr{N}^{*} \vDash \exists^{\infty} Y \phi(\bar{A}, \bar{a}, B, Y)$. By (3) there is an unbounded $D \subseteq N$, a $(1+q)$-term $\rho(u, \bar{v})$, and $d \in N$ such that $\mathscr{N}^{*} \vDash \phi(\bar{A}, \bar{a}, B, D)$ and $D$ satisfies $\langle\rho(d, \bar{v}), m\rangle$ for each $m<\omega$. By the inductive hypothesis, $\mathscr{N}^{*} \vDash \phi_{Y, \rho}(\bar{A}, \bar{a}, B, d)$. From (4) we get $D_{0} \in \operatorname{Class}(\mathscr{N})$ such that $D_{0}$ satisfies $\langle\rho(d, \bar{v}), m(\phi, Y, \rho)\rangle$. Again, by the inductive hypothesis, $\mathcal{N}^{*} \vDash \phi\left(\bar{A}, \bar{a}, B, D_{0}\right)$. Applying the inductive hypothesis one more time yields that $\mathscr{N}^{*} \vDash \phi_{X, \tau}\left(\bar{A}, \bar{a}, b, D_{0}\right)$. Thus, $\mathscr{N}^{*} \vDash$ $\exists^{\infty} Y\left(C(Y) \wedge \phi_{X, \tau}(\bar{A}, \bar{a}, b, Y)\right)$, or in other terms, $\mathscr{N}^{*} \vDash\left(\exists^{\infty} Y \phi\right)_{X, \tau}(\bar{A}, \bar{a}, b, Y)$.

Conversely, suppose $\mathscr{N}^{*} \vDash\left(\exists^{\infty} Y \phi\right)_{X, \tau}(\bar{A}, \bar{a}, b, Y)$, so that

$$
\mathscr{N}^{*} \vDash \exists^{\infty} Y\left(C(Y) \wedge \phi_{X, \tau}(\bar{A}, \bar{a}, b, Y)\right) \text {. }
$$

Thus, there is an unbounded $D \in \operatorname{Class}(\mathscr{N})$ such that $\mathscr{N}^{*} \vDash \phi_{X, \tau}(\bar{A}, \bar{a}, b, D)$. By the inductive hypothesis, $\mathscr{N}^{*} \vDash \phi(\bar{A}, \bar{a}, B, D)$, so that $\mathscr{N}^{*} \vDash \exists^{\infty} Y \phi(\bar{A}, \bar{a}, B, Y)$.

Next, we suppose $\mathscr{N}^{*} \vDash Q^{*} x \in B \phi(x, B)$, where $\phi$ possibly contains some undisplayed first-order parameters. Let $\psi(x, u)$ be a first-order formula such that $\mathscr{N}^{*} \vDash \forall x \forall u\left(\psi \leftrightarrow \phi_{X, \tau}\right)$. Let $d \in N$ be such that for all $a \in B$, if $a>d$ then $\mathscr{N}^{*} \vDash \phi(a, B)$. By the inductive hypothesis, for all $a \in B$, if $a>d$, then $\mathscr{N}^{*} \vDash$ $\phi_{X, \tau}(a, b)$ or, equivalently, $\mathscr{N}^{*} \vDash \psi(a, b)$. From the definition of

$$
m=m\left(Q^{*} x \in X \phi, X, \tau\right),
$$

we see that

$$
\mathscr{N}^{*} \vDash \exists w \forall \bar{v}\left(w<v_{0}<v_{1}<\cdots<v_{s-1} \wedge \bigwedge_{i<s} \theta_{m}\left(v_{i}\right) \rightarrow \psi(\tau(b, \bar{v}), b)\right)
$$


or

$$
\mathscr{N}^{*} \vDash \exists w \forall \bar{v}\left(w<v_{0}<v_{1}<\cdots<v_{s-1} \wedge \bigwedge_{i<s} \theta_{m}\left(v_{i}\right) \rightarrow \neg \psi(\tau(b, \bar{v}), b)\right) .
$$

But since $B$ satisfies $\langle\tau(b, \bar{v}), m\rangle$, the second alternative is impossible. Thus, $\mathscr{N}^{*} \vDash\left(Q^{*} x \in X \phi\right)_{X, \tau}(b)$.

For the converse, just reverse the previous argument.

6. Problems. There are some very natural questions which have been left unanswered in this paper. In this section we mention what seem to be the most interesting of them.

We constructed natural models of various fragments of true second-order arithmetic in \$2. The method of construction of these models depended upon Theorem 2.5 which relied on there being a certain amount of collection in the model. Are there methods of constructing natural models which do not require so much collection? The following is a specific test question.

QuEsTION 6.1. Is there a natural model of CA which is not a model of AC?

Very little seems to be known about the relative strengths of the various $\operatorname{PA}\left(Q^{s}\right)$. For which $s$ and $t$ are there models of $\operatorname{PA}\left(Q^{s}\right)$ which are not models of $\operatorname{PA}\left(Q^{t}\right)$ ? We formally state the most extreme versions of this question.

QuEstion 6.2. Is there a model of $\operatorname{PA}\left(Q^{2}\right)$ which is not a model of $\operatorname{PA}\left(Q^{3}\right)$ or even of $\operatorname{PA}\left(\mathscr{H} \mathscr{R}_{1}\right)$ ?

Question 6.3. Is there a model of $\operatorname{PA}\left(Q^{1,1}\right)$ which is not a model of some $\operatorname{PA}\left(Q^{1,1, \ldots, 1}\right)$ ?

We do know from Corollary 3.9 that there are models of $\operatorname{PA}\left(Q^{1,1}\right)$ which are not models of $\operatorname{PA}\left(Q^{2}\right)$; in fact there is a model of $\operatorname{PA}\left(Q^{1,1}\right)$ which is not $\kappa$-like. Is there another approach to getting such models? To be specific, we ask the following question.

Question 6.4. Is there a model of $\operatorname{PA}\left(Q^{1,1}\right)$ which is not a model of $\operatorname{PA}\left(Q^{2}\right)$ but which is $\kappa$-like for some regular $\kappa$ ?

We applied hyper-Ramsey logic only to Peano arithmetic, but we formulated its definition in Definition 4.1 to be applicable in other situations. For example, we can in general give the quantifier $Q$ the usual $\aleph_{1}$-interpretation and then ask

QUESTION 6.5. Under appropriate set-theoretic hypotheses, are there compactness and/or completeness theorems for $\mathscr{H}_{\mathscr{R}_{\omega}}$ or any of it fragments $\mathscr{H}_{\mathscr{R}_{n}}$ ?

Finally we come to what are probably the major questions left unanswered here. In contrast to the results of [8] concerning $\operatorname{PA}\left(Q^{2}\right)$, we have been unable to obtain either the compactness or completeness of $\operatorname{PA}\left(\mathscr{H} \mathscr{R}_{\omega}\right)$ or any of its fragments.

Question 6.6 (Compactness). Suppose $1 \leqslant n \leqslant \omega$. Is PA $\left(\mathscr{H} \mathscr{R}_{n}\right)$ compact? That is, if $\operatorname{PA}\left(\mathscr{H} \mathscr{R}_{n}\right) \subseteq T \subseteq \mathscr{H} \mathscr{R}_{n}$ and every finite subset of $T$ has a model, then does $T$ have a model?

QUESTION 6.7 (Completeness). Suppose $1 \leqslant n \leqslant \omega$. Is the set of sentences $\sigma \in \mathscr{H}_{R_{n}}$ true in all models of $\operatorname{PA}\left(\mathscr{H} \mathscr{R}_{n}\right)$ recursively enumerable? 


\section{REFERENCES}

1. M. Kaufmann, A rather classless model, Proc. Amer. Math. Soc. 62 (1977), 330-333.

2. K. Kunen, Combinatorics, Handbook of Mathematical Logic, North-Holland, Amsterdam, 1977 , pp. $371-401$.

3. R. MacDowell and E. Specker, Modelle der Arithmetik, Infinitistic Methods, Proc. Sympos. Foundations of Math. (Warsaw, 1959), Pergamon Press, New York, 1961, pp. 257-263.

4. A. Macintyre, Ramsey quantifiers in arithmetic, Model Theory of Algebra and Arithmetic, Lecture Notes in Math., vol. 834, Springer-Verlag, Berlin and New York, 1980, pp. 186-210.

5. M. Magidor and J. I. Malitz, Compact extensions of L(Q) ( Part 1a), Ann. Math. Logic 11 (1977), $217-261$.

6. J. H. Schmerl, Peano models with many generic classes, Pacific J. Math. 46 (1973), 523-536; Correction 92 (1981), 195-198.

7. __ Recursively saturated, rather classless models of Peano arithmetic, Logic Year 1979-1980, Lecture Notes in Math., vol. 859, Springer-Verlag, Berlin and New York, 1981, pp. 268-282.

8. J. H. Schmerl and S. G. Simpson, On the role of the Ramsey quantifiers in first order arithmetic, J. Symbolic Logic 47 (1982), 423-435.

9. J. H. Schmerl, Peano arithmetic and hyper-Ramsey logic, Abstracts Amer. Math. Soc. 3 (1982), 412.

10. W. Sieg, Conservation theorems for subsystems of analysis with restricted induction (abstract), J. Symbolic Logic 46 (1981), 194.

Department of Mathematics, University of Connecticut, Storrs, Connecticut 06268 